\title{
ON THE CONVEX CONES ARISING FROM CLASSIFICATIONS OF PARTIAL ENTANGLEMENT IN THE THREE QUBIT SYSTEM
}

\author{
KYUNG HOON HAN AND SEUNG-HYEOK KYE
}

\begin{abstract}
In order to classify partial entanglement of multi-partite states, it is natural to consider the convex hulls, intersections and differences of basic convex cones obtained from partially separable states with respect to partitions of systems. In this paper, we consider convex cones consisting of $\mathrm{X}$-shaped three qubit states arising in this way. The class of $\mathrm{X}$-shaped states includes important classes like GreenbergerHorne-Zeilinger diagonal states. We find all the extreme rays of those convex cones to exhibit corresponding partially separable states. We also give characterizations for those cones which give rise to necessary criteria in terms of diagonal and anti-diagonal entries for general three qubit states.
\end{abstract}

\section{InTRODUCTION}

The notion of entanglement is now considered as an indispensable resource in the current quantum information theory. In the multi-partite systems, there are various notions of separability according to partitions of systems, which give rise to different kinds of partial entanglement. In the tri-partite system, we may consider three kinds of bi-partitions $A-B C, B-C A$ and $C-A B$ of systems. In this way, a tri-partite state may be considered as a bi-partite state with respect to one of the above bi-partitions. It was shown in [1] that a three qubit state may be entangled even though it is separable as a bi-partite state with respect to any bi-partitions. Therefore, it is natural to classify partial separability in multi-partite systems, as they were suggested in the liturature [2, 3, 4, 5, 6, 7, 8, 9, 10]. We recall that a multi-partite state is (fully) separable if it is a convex sum of pure product states, and entangled if it is not separable.

We will work in the real vector space of all three qubit self-adjoint matrices, and consider the convex cones $\mathcal{A}, \mathcal{B}$ and $\mathcal{C}$ consisting of all unnormalized separable states with respect to the bi-partitions $A-B C, B-C A$ and $C-A B$, respectively. Recall that a subset $C$ of a real vector space is called a convex cone when $C+C \subset C$ and $a C \subset C$ for $a \geq 0$. We note that the sum $C_{1}+C_{2}$ of two convex cones $C_{1}$ and $C_{2}$ is again a convex cone which coincides with the convex hull of $C_{1}$ and $C_{2}$, that is, the smallest convex set containing $C_{1}$ and $C_{2}$.

The above mentioned result [1] tells us that the convex cone $\mathcal{A} \cap \mathcal{B} \cap \mathcal{C}$ is strictly bigger than the convex cone of all fully separable states as tri-partite states. The differences $\mathcal{A} \backslash(\mathcal{B} \cup \mathcal{C})$ and $(\mathcal{A} \cap \mathcal{B}) \backslash \mathcal{C}$ have been considered in [4, 5], together with similar sets obtained by permuting $\mathcal{A}, \mathcal{B}$ and $\mathcal{C}$. On the other hand, the convex hull

1991 Mathematics Subject Classification. 81P40, 52A20, $15 \mathrm{~A} 69$.

Both KHH and SHK were partially supported by NRF-2017R1A2B4006655, Korea. 
$\mathcal{A}+\mathcal{B}+\mathcal{C}$ and the difference $(\mathcal{A}+\mathcal{B}+\mathcal{C}) \backslash(\mathcal{A} \cup \mathcal{B} \cup \mathcal{C})$ have been also considered in [2] and [7, respectively. More recently, all the possible classes

$$
\left[C_{1} \cap \cdots \cap C_{k}\right] \backslash\left[C_{k+1} \cup \cdots \cup C_{\ell}\right]
$$

have been considered in [10], where $C_{i}$ is one of the following convex cones

$$
\mathcal{A}, \quad \mathcal{B}, \quad \mathcal{C}, \quad \mathcal{A}+\mathcal{B}, \quad \mathcal{B}+\mathcal{C}, \quad \mathcal{C}+\mathcal{A}, \quad \mathcal{A}+\mathcal{B}+\mathcal{C} .
$$

Nontrivial classes of three qubit states obtained by (11) are known to be nonempty only recently [11].

The main purposes of this note are twofold: Exhibiting three qubit states in the above classes in (2) and giving criteria for states to be members of the cones. We will do these for so called $\mathrm{X}$-shaped three qubit states, whose entries are zero, by definition, except for diagonal and anti-diagonal entries. Many important states like GHZ diagonal states are of this form. An X-shaped state is also called as an X-state for brevity. In order to exhibit all the three qubit $\mathbf{X}$-states in a given cone, we find all the extreme rays of the convex cone. Recall that an element $x$ of a convex cone $C$ generates an extreme ray whenever $x=x_{1}+x_{2}$ with $x_{i} \in C$ implies that $x_{i}$ is a nonnegative multiple of $x$ for $i=1,2$. By the abuse of the terminology, we say that $x$ itself is an extreme ray in this case. Then all the elements of convex cones in (2) are the nonnegative sums of extreme rays. Those extreme rays also play essential roles to find criteria for the dual cone. Those criteria will be expressed in terms of algebraic inequalities with the entries of $\mathrm{X}$-states, which give rise to necessary criteria for general three qubit states to be a member of a given cone, in terms of diagonal and anti-diagonal entries. As for the corresponding results for full separability, we refer to the papers [12, 13, 14, 15].

Our main tool is the duality between closed convex cones in real vector spaces, and so, we will also consider the dual cones, whose members play roles of witnesses, of the cones in (2). Since the intersection and the convex hull are dual operations, we also naturally consider the intersections as well as convex hulls through the discussion. Therefore, we will consider the convex cones appearing in the following diagram

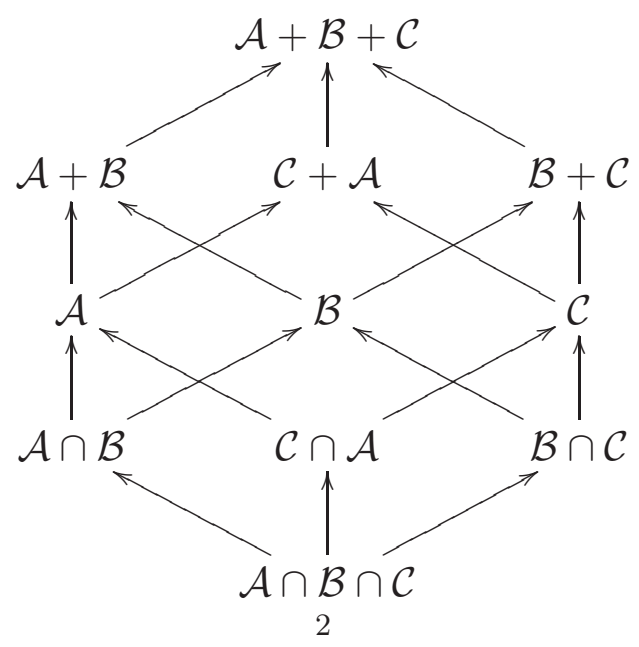


which shows us partial order relations by inclusion among convex cones we are considering. The dual cones will be also discussed.

After we explain briefly the duality in the next section, we will consider the convex cones $\mathcal{A}, \mathcal{B}$ and $\mathcal{C}$ in Section 3 together with their dual cones. We will also consider the convex cones $\mathcal{A}+\mathcal{B}+\mathcal{C}$ and $\mathcal{A} \cap \mathcal{B} \cap \mathcal{C}$ in Section 4. Conditions for those convex cones with $X$-shaped matrices are already scattered in the literature [16, 17, 18, 19, 20]. Here, we give an alternative proof in the context of duality, together with exhibition of all extreme rays in the convex cone of $\mathrm{X}$-shaped matrices in each of them. In Section 5. we deal with convex hulls and intersections of two convex cones like $\mathcal{A}+\mathcal{B}, \mathcal{A} \cap \mathcal{B}$ and their duals. We will summarize our results in the final section.

The authors are grateful to the referee for careful reading and valuable suggestions to improve presentation.

\section{DUALITY}

Let $C$ be a subset of a finite dimensional real vector space $V$ with a non-degenerating bilinear pairing $\langle$,$\rangle , that is, \langle x, y\rangle=0$ for every $y \in V$ implies $x=0$. We define the dual cone $C^{\circ}$ by

$$
C^{\circ}=\{x \in V:\langle x, y\rangle \geq 0 \text { for every } y \in C\} .
$$

Then $C^{\circ}$ is a closed convex cone of $V$ in general, and $C^{\circ \circ}$ is the smallest closed convex cone containing $C$ by the Hahn-Banach type separation theorem. If $C$ itself is a closed convex cone then we have $C=C^{\circ \circ}$, and so we see that the following are equivalent:

- $x \notin C$;

- there exists $y \in C^{\circ}$ such that $\langle x, y\rangle<0$.

For example, if $\mathcal{S}$ is the closed convex cone consisting of unnormalized fully separable states in the real vector space $V$ of self-adjoint matrices in $M_{2} \otimes M_{2} \otimes M_{2}$, then we see by this principle that $\varrho$ is non-separable, that is, entangled if and only if there exists $W \in \mathcal{S}^{\circ}$ such that $\langle W, \varrho\rangle<0$. Such a $W$ must be non-positive, and called an entanglement witness [21]. Here, the bilinear pairing is given by $\langle a, b\rangle=\operatorname{Tr}\left(b a^{\mathrm{t}}\right)$ for

matrices $a$ and $b$, as usual. On the other hand, the closed convex cone $\mathcal{P}$ of all positive matrices is self-dual, that is, $\mathcal{P}^{\circ}=\mathcal{P}$, by the Hadamard theorem.

We note that the two operations, convex hull and intersection, are dual to each other. In other words, the following identities

$$
\left(C_{1}+C_{2}\right)^{\circ}=C_{1}^{\circ} \cap C_{2}^{\circ}, \quad\left(C_{1} \cap C_{2}\right)^{\circ}=C_{1}^{\circ}+C_{2}^{\circ}
$$

hold for closed convex cones $C_{1}$ and $C_{2}$. The first identity follows from the definition. See [22]. The second one follows from the first one and the fact that the convex hull of two closed convex cones is closed. This is an easy consequence of Carathéodory theorem which tells us that the convex hull of a compact set is compact. We note that a convex cone $C$ spans the whole space $V$ if and only if $C+(-C)=V$. If we apply 
the above duality to the four closed convex cones $C,-C,\{0\}$ and $V$, then we see that the following two properties

$\left(\mathrm{C}_{1}\right) C$ spans the whole space;

$\left(\mathrm{C}_{2}\right) C \cap(-C)=\{0\}$

are dual to each other. In other words, a closed convex cone $C$ satisfies $\left(\mathrm{C}_{1}\right)$ if and only if $C^{\circ}$ satisfies $\left(\mathrm{C}_{2}\right)$. Recall that the real vector space $\left(M_{n_{1}} \otimes M_{n_{2}}\right)_{\mathrm{sa}}$ of all selfadjoint matrices in the tensor product $M_{n_{1}} \otimes M_{n_{2}}$ coincides with the tensor product $\left(M_{n_{1}}\right)_{\mathrm{sa}} \otimes\left(M_{n_{2}}\right)_{\mathrm{sa}}$ of the self-adjoint parts. See [15, Section 7]. This is also true for multi-tensor products by induction. Therefore, the convex cone $\mathcal{S}=M_{2}^{+} \otimes M_{2}^{+} \otimes M_{2}^{+}$ spans the whole space $V=\left(M_{2} \otimes M_{2} \otimes M_{2}\right)_{\text {sa }}$. Since $\mathcal{P}=\left(M_{2} \otimes M_{2} \otimes M_{2}\right)^{+}$satisfies $\mathcal{P} \cap(-\mathcal{P})=\{0\}$, we see that all the convex cones $\mathcal{X}$ in the diagram (3) also satisfy both conditions, by the relation $\mathcal{S} \subset \mathcal{X} \subset \mathcal{P}$. We list up the dual cones of the cones in (3) as follows:

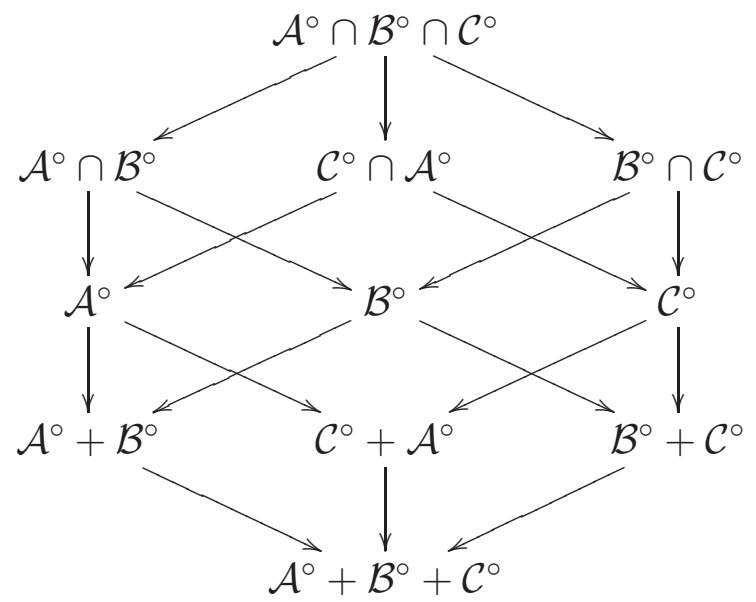

We note that all the convex cones in the diagram (4) also satisfy both conditions $\left(\mathrm{C}_{1}\right)$ and $\left(\mathrm{C}_{2}\right)$, as dual cones of the convex cones satisfying the conditions. An important consequence of $\left(\mathrm{C}_{2}\right)$ is that every element of the convex cone is a nonnegative sum of extreme rays. See [23, Theorem 18.5].

The duality is also very useful to find all the candidates for extreme rays. We say that a subset $S$ of a closed convex cone $C$ is a generating set for $C$ if every element of $C$ is the limit of nonnegative sums of finitely many elements in $S$. This happens if and only if $S^{\circ \circ}=C$ if and only if $S^{\circ}=C^{\circ}$. In other words, we have to show that the following two statements

- $y \in C^{\circ}$, that is, $\langle x, y\rangle \geq 0$ for each $x \in C$;

- $\langle x, y\rangle \geq 0$ for each $x \in S$

are equivalent to each other, in order to show that $S$ is a generating set for $C$. This equivalence, in turn, gives rise to a criterion for the convex cone $C^{\circ}$ in terms of algebraic 
inequalities arising from members in the generating set $S$. This principle will be the main tool of our discussion throughout this paper.

We note that generating sets of a convex cone are not determined uniquely. For example, the convex cone $C$ itself is also a generating set for $C$. Furthermore, a generating set need not contain all the extreme rays. If a generating set $S$ for $C$ is closed, then its convex hull is also closed by Carathéodory theorem, and so every element of $C$ is the sum of finitely many elements in $S$. Therefore, we conclude that a closed generating set for $C$ contains all the extreme rays of $C$. In this way, we are looking for the set $\operatorname{Ext}(C)$ of all extreme rays of the convex cone $C$. We summarize as follows:

Proposition 2.1. For a subset $S$ of a closed convex cone $C$ in a finite dimensional real vector space $V$, the following are equivalent:

(i) $S$ is a generating set for $C$;

(ii) if $y \in V$ and $\langle x, y\rangle \geq 0$ for each $x \in S$, then $y \in C^{\circ}$.

If $S$ is a closed generating set for $C$, then we have $\operatorname{Ext}(C) \subset S$.

In this paper, we will concentrate on the three qubit system, and so we will work in the real vector space $V$ of all $8 \times 8$ self-adjoint matrices. The space $V$ has an important subspace, denoted by $\mathbf{X}$, consisting of all $\mathbf{X}$-shaped matrices whose entries are zero except for diagonal and anti-diagonal entries. In the three qubit case, an $\mathbf{X}$-shaped self-adjoint matrix is of the form

$$
\mathrm{X}(a, b, z)=\left(\begin{array}{ccccccccc}
a_{1} & & & & & & & z_{1} \\
& a_{2} & & & & & z_{2} & \\
& & a_{3} & & & z_{3} & & \\
& & & a_{4} & z_{4} & & & \\
& & & \bar{z}_{4} & b_{4} & & & \\
& & \bar{z}_{3} & & & b_{3} & & \\
& \bar{z}_{2} & & & & & b_{2} & \\
\bar{z}_{1} & & & & & & & b_{1}
\end{array}\right),
$$

for $a, b \in \mathbb{R}^{4}$ and $z \in \mathbb{C}^{4}$, where $\mathbb{C}^{2} \otimes \mathbb{C}^{2} \otimes \mathbb{C}^{2}$ is identified with the space $\mathbb{C}^{8}$ using the lexicographic order of indices. Many important multi-qubit states arise in this form. For example, GHZ diagonal states [24] are in this form, and an $\mathbf{X}$-state $\mathbf{X}(a, b, z)$ is a GHZ diagonal if and only if $a=b$ and $z \in \mathbb{R}^{4}$.

Note that $V$ and $\mathrm{X}$ are of 64 and 16-dimensional spaces, respectively. For a given matrix $\varrho \in V$, we denote by $\varrho$ x the $\mathrm{X}$-part of $\varrho$. The map $\varrho \mapsto \varrho$ from $V$ onto $\mathrm{X}$ has the following important property.

Proposition 2.2. For every convex cone $\mathcal{X}$ in the diagram (3), we have the following:

(i) if $\varrho \in \mathcal{X}$, then $\varrho x \in \mathcal{X}$;

(ii) if $W \in \mathcal{X}^{\circ}$, then $W_{\mathrm{X}} \in \mathcal{X}^{\circ}$. 
Proof. It suffices to prove for the convex cone $\mathcal{A}$. For the statement (i), it also suffices to show for a vector state $\varrho$ associated with a product vector $|x\rangle \otimes|y\rangle \in \mathbb{C}^{2} \otimes \mathbb{C}^{4}$, where $|x\rangle=\left(x_{1}, x_{2}\right)^{\mathrm{t}}$ and $|y\rangle=\left(y_{1}, y_{2}, y_{3}, y_{4}\right)^{\mathrm{t}}$. We consider the following product vectors

$$
\begin{aligned}
& \left(+x_{1},+x_{2}\right)^{\mathrm{t}} \otimes\left(+y_{1},+y_{2},+y_{3},+y_{4}\right)^{\mathrm{t}}, \\
& \left(+x_{1},+x_{2}\right)^{\mathrm{t}} \otimes\left(+y_{1},-y_{2},-y_{3},+y_{4}\right)^{\mathrm{t}}, \\
& \left(+x_{1},-x_{2}\right)^{\mathrm{t}} \otimes\left(+y_{1},-y_{2},+y_{3},-y_{4}\right)^{\mathrm{t}}, \\
& \left(+x_{1},-x_{2}\right)^{\mathrm{t}} \otimes\left(+y_{1},+y_{2},-y_{3},-y_{4}\right)^{\mathrm{t}} .
\end{aligned}
$$

We take the average of four vector states associated with these four product vectors, to recover the $\mathrm{X}$-part of $\varrho$. This proves (i). For the statement (ii) with $\mathcal{X}=\mathcal{A}$, take $W \in \mathcal{A}^{\circ}$. For every $\varrho \in \mathcal{A}$, we see that $\left\langle W_{\mathrm{x}}, \varrho\right\rangle=\langle W, \varrho \mathbf{x}\rangle$ is nonnegative, because $W \in \mathcal{A}^{\circ}$ and $\varrho_{\mathrm{x}} \in \mathcal{A}$ by (i). Therefore, we have $W_{\mathrm{x}} \in \mathcal{A}^{\circ}$.

Corresponding results for full separability are found in Section 3 of [12]. See also Proposition 4.1 of [15] for multi-qubit cases. If $\varrho$ is an X-state, then $\langle W, \varrho\rangle=\left\langle W_{\mathrm{X}}, \varrho\right\rangle$, and so we have the following:

Corollary 2.3. For a convex cone $\mathcal{X}$ in the diagram (3), we have the following:

(i) for a three qubit $\mathbf{X}$-state $\varrho$, we have $\varrho \in \mathcal{X}$ if and only if $\langle W, \varrho\rangle \geq 0$ for every $\mathrm{X}$-shaped $W \in \mathcal{X}^{\circ}$;

(ii) for a three qubit $\mathbf{X}$-shaped $W$, we have $W \in \mathcal{X}^{\circ}$ if and only if $\langle W, \varrho\rangle \geq 0$ for every $\mathrm{X}$-state $\varrho \in \mathcal{X}$.

Corollary 2.4. For convex cones $\mathcal{X}_{1}$ and $\mathcal{X}_{2}$ in diagrams (3) or (41), we have the relation $\left(\mathcal{X}_{1}+\mathcal{X}_{2}\right) \cap \mathrm{X}=\left(\mathcal{X}_{1} \cap \mathrm{X}\right)+\left(\mathcal{X}_{2} \cap \mathrm{X}\right)$.

Once we characterize X-shaped matrices in the convex cones in (33) or (44), these conditions will give rise to necessary conditions for general three qubit self-adjoint matrices to belong to those convex cones, by Proposition 2.2. On the other hand, Corollary 2.3 tells us that we may restrict ourselves on the bi-linear pairing in the real vector space $\mathbf{X}$ for this purpose.

\section{BASIC PARTIAL SEPARABILITY}

In this section, we consider the three basic convex cones $\mathcal{A}, \mathcal{B}, \mathcal{C}$ and their dual cones $\mathcal{A}^{\circ}, \mathcal{B}^{\circ}, \mathcal{C}^{\circ}$. It was shown in [20, Proposition 5.2] that an X-shaped multi-qubit state is separable with respect to a bi-partition of systems if and only if it is of positive partial transpose with respect to the same bi-partition. The PPT condition is easily checked for three qubit $\mathrm{X}$-shaped states by the following inequalities

$$
S_{1}[i, j]: \quad \min \left\{\sqrt{a_{i} b_{i}}, \sqrt{a_{j} b_{j}}\right\} \geq \max \left\{\left|z_{i}\right|,\left|z_{j}\right|\right\}
$$


for $i, j=1,2,3,4$. If $\varrho=\mathrm{X}\left(a, b,\left(z_{1}, z_{2}, z_{3}, z_{4}\right)\right)$, then the partial transposes are given by

$$
\begin{aligned}
& \varrho^{\Gamma_{A}}=\mathrm{X}\left(a, b,\left(\bar{z}_{4}, \bar{z}_{3}, \bar{z}_{2}, \bar{z}_{1}\right)\right), \\
& \varrho^{\Gamma_{B}}=\mathrm{X}\left(a, b,\left(z_{3}, z_{4}, z_{1}, z_{2}\right)\right), \\
& \varrho^{\Gamma_{C}}=\mathrm{X}\left(a, b,\left(z_{2}, z_{1}, z_{4}, z_{3}\right)\right) .
\end{aligned}
$$

Therefore, we have the following:

Proposition 3.1. [20, Proposition 5.2] For a three qubit $\mathbf{X}$-state $\varrho=\mathrm{X}(a, b, z)$, we have the following:

(i) $\varrho \in \mathcal{A}$ if and only if both $S_{1}[1,4]$ and $S_{1}[2,3]$ hold;

(ii) $\varrho \in \mathcal{B}$ if and only if both $S_{1}[1,3]$ and $S_{1}[2,4]$ hold;

(iii) $\varrho \in \mathcal{C}$ if and only if both $S_{1}[1,2]$ and $S_{1}[3,4]$ hold.

We note that inequalities $S_{1}[i, j]$ 's give us necessary criteria for general three qubit states to belong to $\mathcal{A}, \mathcal{B}$ and $\mathcal{C}$, respectively, by Proposition 2.2. Now, we proceed to provide generating sets for the convex cones $\mathcal{A} \cap \mathrm{X}, \mathcal{B} \cap \mathrm{X}$ and $\mathcal{C} \cap \mathrm{X}$. To be motivated, we decompose an $\mathrm{X}$-state $\varrho=\mathrm{X}(a, b, z)$ in $\mathcal{A}$ by

$$
\begin{aligned}
\varrho=\mathrm{X}\left(\left(a_{1}, 0,0, a_{4}\right),\right. & \left.\left(b_{1}, 0,0, b_{4}\right),\left(z_{1}, 0,0, z_{4}\right)\right) \\
& +\mathrm{X}\left(\left(0, a_{2}, a_{3}, 0\right),\left(0, b_{2}, b_{3}, 0\right),\left(0, z_{2}, z_{3}, 0\right)\right),
\end{aligned}
$$

then two summands satisfy both $S_{1}[1,4]$ and $S_{1}[2,3]$. Therefore, we may assume that $a_{k}=b_{k}=z_{k}=0$ for $k=2,3$. If $z_{1}=0$, then $\varrho$ is the average of two states

$$
\mathrm{X}\left(\left(a_{1}, 0,0, a_{4}\right),\left(b_{1}, 0,0, b_{4}\right),\left(z_{4}, 0,0, z_{4}\right)\right), \quad \mathrm{X}\left(\left(a_{1}, 0,0, a_{4}\right),\left(b_{1}, 0,0, b_{4}\right),\left(-z_{4}, 0,0, z_{4}\right)\right)
$$

in $\mathcal{A} \cap \mathrm{X}$. If $0<\left|z_{1}\right|<\left|z_{4}\right|$, then $\varrho$ is a convex combination of

$\mathbf{X}\left(\left(a_{1}, 0,0, a_{4}\right),\left(b_{1}, 0,0, b_{4}\right),\left(\frac{\left|z_{4}\right|}{\left|z_{1}\right|} z_{1}, 0,0, z_{4}\right)\right), \quad \mathbf{X}\left(\left(a_{1}, 0,0, a_{4}\right),\left(b_{1}, 0,0, b_{4}\right),\left(-\frac{\left|z_{4}\right|}{\left|z_{1}\right|} z_{1}, 0,0, z_{4}\right)\right)$ in $\mathcal{A} \cap \mathrm{X}$.

By subtracting a suitable diagonal state, it is natural to consider the following conditions

$$
S_{1}^{e}[i, j]: \quad \sqrt{a_{i} b_{i}}=\left|z_{i}\right|=\sqrt{a_{j} b_{j}}=\left|z_{j}\right|=1, \quad \text { the others are zero, }
$$

for each $i, j=1,2,3,4$ with $i \neq j$. We define

$$
\begin{aligned}
& \mathcal{E}_{\mathcal{A}}=\left\{\varrho=\mathrm{X}(a, b, z): S_{1}^{e}[1,4] \text { or } S_{1}^{e}[2,3] \text { holds }\right\}, \\
& \mathcal{E}_{\mathcal{B}}=\left\{\varrho=\mathrm{X}(a, b, z): S_{1}^{e}[1,3] \text { or } S_{1}^{e}[2,4] \text { holds }\right\}, \\
& \mathcal{E}_{\mathcal{C}}=\left\{\varrho=\mathrm{X}(a, b, z): S_{1}^{e}[1,2] \text { or } S_{1}^{e}[3,4] \text { holds }\right\} .
\end{aligned}
$$

We also denote by $\Delta$ the set of all extreme diagonal states, that is,

$$
\Delta=\left\{\mathrm{X}\left(E_{i}, 0,0\right): i=1,2,3,4\right\} \cup\left\{\mathrm{X}\left(0, E_{i}, 0\right): i=1,2,3,4\right\},
$$

where $\left\{E_{i}: i=1,2,3,4\right\}$ denotes the canonical basis of $\mathbb{R}^{4}$.

We have $\mathcal{E}_{\mathcal{A}} \subset \mathcal{A}$ by Proposition 3.1 , and $\mathcal{E}_{\mathcal{A}}$ is parameterized by four real variables. The same comments also hold for $\mathcal{B}$ and $\mathcal{C}$. We also consider the following inequalities: 


$$
W_{1}[i, j]: \quad \sqrt{s_{i} t_{i}}+\sqrt{s_{j} t_{j}} \geq\left|u_{i}\right|+\left|u_{j}\right|
$$

for $i, j=1,2,3,4$ with $i \neq j$, in order to characterize the dual cones $\mathcal{A}^{\circ} \cap \mathrm{X}, \mathcal{B}^{\circ} \cap \mathbf{X}$ and $\mathcal{C}^{\circ} \cap \mathrm{X}$. We denote $\mathbf{X}_{i}\left(s_{i}, t_{i}, u_{i}\right):=\mathbf{X}\left(s_{i} E_{i}, t_{i} E_{i}, u_{i} E_{i}\right)$ for $i=1,2,3,4$.

Lemma 3.2. For a given self-adjoint $\mathrm{X}$-shaped matrix $W=\mathrm{X}(s, t, u)$, the following are equivalent:

(i) $\langle W, \varrho\rangle \geq 0$ for each $\varrho \in \mathcal{E}_{\mathcal{A}} \cup \Delta$;

(ii) $s_{i}, t_{i} \geq 0$ for $i=1,2,3,4$, and the inequalities $W_{1}[1,4]$ and $W_{1}[2,3]$ hold;

(iii) $\langle W, \varrho\rangle \geq 0$ for each $\varrho \in \mathcal{A}$.

Proof. For the direction (i) $\Longrightarrow$ (ii), we obtain $s_{i}, t_{i} \geq 0$ from $\langle W, \varrho\rangle \geq 0$ for $\varrho \in \Delta$. Suppose that both $s_{i}$ and $t_{i}$ are nonzero for each $i=1,2,3,4$. Then, we can consider the following states

$$
\varrho_{i, j}:=\mathrm{X}_{i}\left(\sqrt{\frac{t_{i}}{s_{i}}}, \sqrt{\frac{s_{i}}{t_{i}}},-e^{-\mathrm{i} \theta_{i}}\right)+\mathrm{X}_{j}\left(\sqrt{\frac{t_{j}}{s_{j}}}, \sqrt{\frac{s_{j}}{t_{j}}},-e^{-\mathrm{i} \theta_{j}}\right)
$$

for $(i, j)=(1,4),(2,3)$, with $\theta_{k}=\arg u_{k}$. Since $\varrho_{i, j} \in \mathcal{E}_{\mathcal{A}}$, we have

$$
0 \leq \frac{1}{2}\left\langle W, \varrho_{i, j}\right\rangle=\sqrt{s_{i} t_{i}}+\sqrt{s_{j} t_{j}}-\left|u_{i}\right|-\left|u_{j}\right|,
$$

by (i). When one of $s_{i}$ or $t_{i}$ is zero, we apply the result to $W+\varepsilon I$ to get the inequality $\sqrt{\left(s_{i}+\varepsilon\right)\left(t_{i}+\varepsilon\right)}+\sqrt{\left(s_{j}+\varepsilon\right)\left(t_{j}+\varepsilon\right)} \geq\left|u_{i}\right|+\left|u_{j}\right|$ for each $\varepsilon>0$.

For the implication (ii) $\Longrightarrow$ (iii), it is enough to prove the following by Corollary 2.3 and Proposition 3.1;

$$
S_{1}[1,4], S_{1}[2,3], W_{1}[1,4], W_{1}[2,3] \Longrightarrow\langle\mathrm{X}(s, t, u), \mathrm{X}(a, b, z)\rangle \geq 0 .
$$

Indeed, we have

$$
\begin{aligned}
\sum_{i=1}^{4} \sqrt{s_{i} t_{i}} \sqrt{a_{i} b_{i}} & =\left(\sqrt{s_{1} t_{1}} \sqrt{a_{1} b_{1}}+\sqrt{s_{4} t_{4}} \sqrt{a_{4} b_{4}}\right)+\left(\sqrt{s_{2} t_{2}} \sqrt{a_{2} b_{2}}+\sqrt{s_{3} t_{3}} \sqrt{a_{3} b_{3}}\right) \\
& \geq\left(\sqrt{s_{1} t_{1}}+\sqrt{s_{4} t_{4}}\right) \max \left\{\left|z_{1}\right|,\left|z_{4}\right|\right\}+\left(\sqrt{s_{2} t_{2}}+\sqrt{s_{3} t_{3}}\right) \max \left\{\left|z_{2}\right|,\left|z_{3}\right|\right\} \\
& \geq\left(\left|u_{1}\right|+\left|u_{4}\right|\right) \max \left\{\left|z_{1}\right|,\left|z_{4}\right|\right\}+\left(\left|u_{2}\right|+\left|u_{3}\right|\right) \max \left\{\left|z_{2}\right|,\left|z_{3}\right|\right\} \\
& \geq \sum_{i=1}^{4}\left|u_{i}\right|\left|z_{i}\right|,
\end{aligned}
$$

which implies

$$
\begin{aligned}
\frac{1}{2}\langle\mathbf{X}(s, t, u), \mathbf{X}(a, b, z)\rangle & =\frac{1}{2} \sum_{i=1}^{4}\left(s_{i} a_{i}+t_{i} b_{i}+2 \operatorname{Re}\left(u_{i} z_{i}\right)\right) \\
& \geq \sum_{\substack{i=1 \\
8}}^{4}\left(\sqrt{s_{i} t_{i}} \sqrt{a_{i} b_{i}}-\left|u_{i}\right|\left|z_{i}\right|\right) \geq 0 .
\end{aligned}
$$


as it was required. The direction (iii) $\Longrightarrow$ (i) is clear since $\mathcal{E}_{\mathcal{A}} \cup \Delta \subset \mathcal{A}$ by Proposition 3.1 .

The equivalence between (ii) and (iii) of Lemma 3.2 gives rise to a characterization of the convex cone $\mathcal{A}^{\circ} \cap \mathrm{X}$, whose members are the Choi matrix of $(1,2,2)$-positive bilinear maps between $2 \times 2$ matrices in the sense of [19]. Therefore, Lemma 3.2 recovers Theorem 6.2 in [19], as follows:

Proposition 3.3. [19, Theorem 6.2] For a self-adjoint $W=\mathrm{X}(s, t, u)$ with nonnegative diagonals, we have the following:

(i) $W \in \mathcal{A}^{\circ}$ if and only if both $W_{1}[1,4]$ and $W_{1}[2,3]$ hold;

(ii) $W \in \mathcal{B}^{\circ}$ if and only if both $W_{1}[1,3]$ and $W_{1}[2,4]$ hold;

(iii) $W \in \mathcal{C}^{\circ}$ if and only if both $W_{1}[1,2]$ and $W_{1}[3,4]$ hold.

The implication (i) $\Longrightarrow$ (iii) of Lemma 3.2 tells us that the set $\mathcal{E}_{\mathcal{A}} \cup \Delta$ is a generating set for the convex cone $\mathcal{A} \cap \mathrm{X}$ by Proposition 2.1. We also note that the set $\mathcal{E}_{\mathcal{A}} \cup \Delta$ is closed, and so we conclude that every extreme ray of $\mathcal{A} \cap \mathrm{X}$ must be an element of $\mathcal{E}_{\mathcal{A}} \cup \Delta$. We show that the converse actually holds. Because states in $\Delta$ generate extreme rays in the cone $\mathcal{P}$, they also generate extreme rays of the smaller convex cones listed in the diagram (3). In order to prove that every state in the set $\mathcal{E}_{\mathcal{A}}$ generates an extreme ray of the convex cone $\mathcal{A} \cap \mathrm{X}$, we first prove a technical lemma which will play a key role in characterization of extreme rays of the other cones.

Lemma 3.4. Suppose that a three qubit $\mathrm{X}$-state $\varrho=\mathrm{X}(a, b, z)$ in $\mathcal{A}$ (respectively, $\mathcal{B}$ and $\mathcal{C}$ ) is decomposed as

$$
\varrho=\mathrm{X}\left(a^{\prime}, b^{\prime}, z^{\prime}\right)+\mathrm{X}\left(a^{\prime \prime}, b^{\prime \prime}, z^{\prime \prime}\right)
$$

in $\mathcal{A} \cap \mathrm{X}$ (respectively, $\mathcal{B} \cap \mathrm{X}$ and $\mathcal{C} \cap \mathrm{X})$. If

$$
\sqrt{a_{i} b_{i}}=\sqrt{a_{j} b_{j}}=\left|z_{i}\right|
$$

for $\{i, j\}=\{1,4\}$ or $\{2,3\}$ (respectively, $\{i, j\}=\{1,3\}$ or $\{2,4\}$, and $\{i, j\}=\{1,2\}$ or $\{3,4\})$, then we have

$$
\left(a_{i}^{\prime}, a_{j}^{\prime}, b_{i}^{\prime}, b_{j}^{\prime}, z_{i}^{\prime}\right) / /\left(a_{i}^{\prime \prime}, a_{j}^{\prime \prime}, b_{i}^{\prime \prime}, b_{j}^{\prime \prime}, z_{i}^{\prime \prime}\right) .
$$

Proof. Let $k=i, j$. We have

$$
\left|z_{i}\right|=\left|z_{i}^{\prime}+z_{i}^{\prime \prime}\right| \leq\left|z_{i}^{\prime}\right|+\left|z_{i}^{\prime \prime}\right| \leq \sqrt{a_{k}^{\prime}} \sqrt{b_{k}^{\prime}}+\sqrt{a_{k}^{\prime \prime}} \sqrt{b_{k}^{\prime \prime}} \leq \sqrt{a_{k}^{\prime}+a_{k}^{\prime \prime}} \sqrt{b_{k}^{\prime}+b_{k}^{\prime \prime}}=\sqrt{a_{k} b_{k}}
$$

by $S_{1}[i, j]$ and the Cauchy-Schwartz inequality. Since $\left|z_{i}\right|=\sqrt{a_{k} b_{k}}$, we have

$$
z_{i}^{\prime} / / z_{i}^{\prime \prime}, \quad \sqrt{a_{k}^{\prime} b_{k}^{\prime}}=\left|z_{i}^{\prime}\right|, \quad \sqrt{a_{k}^{\prime \prime} b_{k}^{\prime \prime}}=\left|z_{i}^{\prime \prime}\right|, \quad\left(\sqrt{a_{k}^{\prime}}, \sqrt{a_{k}^{\prime \prime}}\right) / /\left(\sqrt{b_{k}^{\prime}}, \sqrt{b_{k}^{\prime \prime}}\right) .
$$

Let $\left(\sqrt{a_{k}^{\prime}}, \sqrt{a_{k}^{\prime \prime}}\right)=\lambda_{k}\left(\sqrt{b_{k}^{\prime}}, \sqrt{b_{k}^{\prime \prime}}\right)$ for $\lambda_{k}>0$. Then we have

$$
\frac{z_{i}^{\prime \prime}}{z_{i}^{\prime}}=\frac{\left|z_{i}^{\prime \prime}\right|}{\left|z_{i}^{\prime}\right|}=\frac{\sqrt{a_{k}^{\prime \prime} b_{k}^{\prime \prime}}}{\sqrt{a_{k}^{\prime} b_{k}^{\prime}}}=\frac{\lambda_{k} b_{k}^{\prime \prime}}{\lambda_{k} b_{k}^{\prime}}=\frac{b_{k}^{\prime \prime}}{b_{k}^{\prime}}=\frac{a_{k}^{\prime \prime} / \lambda_{k}^{2}}{a_{k}^{\prime} / \lambda_{k}^{2}}=\frac{a_{k}^{\prime \prime}}{a_{k}^{\prime}},
$$

as it was required. 
Theorem 3.5. We have

$$
\operatorname{Ext}(\mathcal{A} \cap \mathrm{X})=\mathcal{E}_{\mathcal{A}} \cup \Delta, \quad \operatorname{Ext}(\mathcal{B} \cap \mathrm{X})=\mathcal{E}_{\mathcal{B}} \cup \Delta \quad \text { and } \quad \operatorname{Ext}(\mathcal{C} \cap \mathrm{X})=\mathcal{E}_{\mathcal{C}} \cup \Delta .
$$

Proof. It suffices to show that every state in the set $\mathcal{E}_{\mathcal{A}}$ generates an extreme ray of the convex cone $\mathcal{A} \cap \mathrm{X}$. Suppose that $\varrho=\mathrm{X}(a, b, z)$ satisfies the condition $S_{1}^{e}[1,4]$ and

$$
\varrho=\mathrm{X}\left(a^{\prime}, b^{\prime}, z^{\prime}\right)+\mathrm{X}\left(a^{\prime \prime}, b^{\prime \prime}, z^{\prime \prime}\right) \quad \text { in } \mathcal{A} \cap \mathrm{X} .
$$

For $j=2,3$, we see that $a_{j}=b_{j}=0$ implies $a_{j}^{\prime}=b_{j}^{\prime}=z_{j}^{\prime}=a_{j}^{\prime \prime}=b_{j}^{\prime \prime}=z_{j}^{\prime \prime}=0$. Applying Lemma 3.4 with $(i, j)=(1,4)$ and $(i, j)=(4,1)$, we get $\left(a^{\prime}, b^{\prime}, z^{\prime}\right) / /\left(a^{\prime \prime}, b^{\prime \prime}, z^{\prime \prime}\right)$, as it was required. The same argument works for the case of $S_{1}^{e}[2,3]$.

In the remainder of this section, we look for extreme rays of $\mathcal{A}^{\circ} \cap \mathrm{X}, \mathcal{B}^{\circ} \cap \mathrm{X}$ and $\mathcal{C}^{\circ} \cap \mathrm{X}$. To do this, we consider the condition

$$
W_{1}^{e}[i, j]: \quad \sqrt{s_{i} t_{i}}=\left|u_{j}\right|=1, \quad \text { the others are zero, }
$$

for $i \neq j$, and define

$$
\begin{aligned}
& \mathcal{E}_{\mathcal{A}^{\circ}}=\left\{W=\mathrm{X}(s, t, u): W_{1}^{e}[i, j] \text { holds for some }(i, j)=(1,4),(4,1),(2,3),(3,2)\right\}, \\
& \mathcal{E}_{\mathcal{B}^{\circ}}=\left\{W=\mathrm{X}(s, t, u): W_{1}^{e}[i, j] \text { holds for some }(i, j)=(1,3),(3,1),(2,4),(4,2)\right\}, \\
& \mathcal{E}_{\mathcal{C}^{\circ}}=\left\{W=\mathrm{X}(s, t, u): W_{1}^{e}[i, j] \text { holds for some }(i, j)=(1,2),(2,1),(3,4),(4,3)\right\} .
\end{aligned}
$$

We also consider the following set

$$
\mathcal{W}^{\Delta}=\left\{W=\mathrm{X}_{i}\left(r, r^{-1}, e^{\mathrm{i} \theta}\right): i=1,2,3,4, r>0, \theta \in \mathbb{R}\right\} .
$$

Lemma 3.6. For a given self-adjoint $\mathbf{X}$-shaped matrix $\varrho=\mathrm{X}(a, b, z)$, the following are equivalent:

(i) $\langle W, \varrho\rangle \geq 0$ for each $W \in \mathcal{E}_{\mathcal{A}^{\circ}} \cup \Delta \cup \mathcal{W}^{\Delta}$;

(ii) $\varrho$ is a state satisfying the inequalities $S_{1}[1,4]$ and $S_{1}[2,3]$;

(iii) $\langle W, \varrho\rangle \geq 0$ for each $W \in \mathcal{A}^{\circ}$.

Proof. The equivalence between (ii) and (iii) follows from Proposition 3.1. Therefore, it suffices to show the direction (i) $\Longrightarrow$ (ii). Since $\langle W, \varrho\rangle \geq 0$ for $W \in \Delta$, we have $a_{i}, b_{i} \geq 0$. By taking $\varrho+\varepsilon I$ into account as in the proof of Lemma 3.2, we may assume that $a_{i}, b_{i}>0$ without loss of generality. Then, we can consider

$$
W_{i, j}:=\mathrm{X}_{i}\left(\sqrt{\frac{b_{i}}{a_{i}}}, \sqrt{\frac{a_{i}}{b_{i}}}, 0\right)+\mathrm{X}_{j}\left(0,0,-e^{\mathrm{i} \theta_{j}}\right) \in \mathcal{E}_{\mathcal{A}^{\circ}} \cup \mathcal{W}^{\Delta}
$$

for $(i, j)=(1,4),(4,1),(2,3),(3,2)$ or $i=j$. Note that $W_{i, i} \in \mathcal{W}^{\Delta}$. We see that $\varrho$ is a state by $\left\langle W_{i, i}, \varrho\right\rangle \geq 0$, and the inequalities $S_{1}[1,4]$ and $S_{1}[2,3]$ follow from $\left\langle W_{i, j}, \varrho\right\rangle \geq 0$ for $(i, j)=(1,4),(4,1),(2,3),(3,2)$.

As for extreme rays of the dual cones, we also begin with a technical lemma which is a witness counterpart to Lemma 3.4 . 
Lemma 3.7. Suppose that a three qubit self-adjoint $\mathrm{X}$-shaped matrix $W=\mathrm{X}(s, t, u)$ in $\mathcal{A}^{\circ}$ (respectively, $\mathcal{B}^{\circ}$ and $\mathcal{C}^{\circ}$ ) is decomposed as

$$
W=\mathrm{X}\left(s^{\prime}, t^{\prime}, u^{\prime}\right)+\mathrm{X}\left(s^{\prime \prime}, t^{\prime \prime}, u^{\prime \prime}\right)
$$

in $\mathcal{A}^{\circ} \cap \mathrm{X}$ (respectively, $\mathcal{B}^{\circ} \cap \mathrm{X}$ and $\left.\mathcal{C}^{\circ} \cap \mathrm{X}\right)$. If

$$
\sqrt{s_{i} t_{i}}=\left|u_{k}\right| \quad \text { and } \quad s_{j}=t_{j}=0
$$

for $\{i, j\}=\{1,4\}$ or $\{2,3\}$ (respectively, $\{i, j\}=\{1,3\}$ or $\{2,4\}$, and $\{i, j\}=\{1,2\}$ or $\{3,4\})$ and $k \in\{i, j\}$, then we have

$$
s_{j}^{\prime}=t_{j}^{\prime}=0=s_{j}^{\prime \prime}=t_{j}^{\prime \prime}, \quad u_{\ell}^{\prime}=0=u_{\ell}^{\prime \prime} \quad \text { and } \quad\left(s_{i}^{\prime}, t_{i}^{\prime}, u_{k}^{\prime}\right) / /\left(s_{i}^{\prime \prime}, t_{i}^{\prime \prime}, u_{k}^{\prime \prime}\right)
$$

for $\ell$ with $\{k, \ell\}=\{i, j\}$.

Proof. The condition $s_{j}=t_{j}=0$ implies $s_{j}^{\prime}=t_{j}^{\prime}=0=s_{j}^{\prime \prime}=t_{j}^{\prime \prime}$. We have

$$
\begin{aligned}
\left|u_{k}\right| & =\left|u_{k}^{\prime}+u_{k}^{\prime \prime}\right| \\
& \leq\left|u_{k}^{\prime}\right|+\left|u_{k}^{\prime \prime}\right| \\
& \leq \sqrt{s_{i}^{\prime}} \sqrt{t_{i}^{\prime}}-\left|u_{\ell}^{\prime}\right|+\sqrt{s_{i}^{\prime \prime}} \sqrt{t_{i}^{\prime \prime}}-\left|u_{\ell}^{\prime \prime}\right| \\
& \leq \sqrt{s_{i}^{\prime}+s_{i}^{\prime \prime}} \sqrt{t_{i}^{\prime}+t_{i}^{\prime \prime}}-\left|u_{\ell}^{\prime}\right|-\left|u_{\ell}^{\prime \prime}\right| \\
& =\sqrt{s_{i} t_{i}}-\left|u_{\ell}^{\prime}\right|-\left|u_{\ell}^{\prime \prime}\right| \\
& \leq \sqrt{s_{i} t_{i}}
\end{aligned}
$$

by $W_{1}[i, j]=W_{1}[k, \ell]$ and the Cauchy-Schwartz inequality. Since $\left|u_{k}\right|=\sqrt{s_{i} t_{i}}$, we have

$$
u_{k}^{\prime} / / u_{k}^{\prime \prime}, \quad u_{\ell}^{\prime}=0=u_{\ell}^{\prime \prime} \quad \sqrt{s_{i}^{\prime} t_{i}^{\prime}}=\left|u_{k}^{\prime}\right|, \quad \sqrt{s_{i}^{\prime \prime} t_{i}^{\prime \prime}}=\left|u_{k}^{\prime \prime}\right|, \quad\left(\sqrt{s_{i}^{\prime}}, \sqrt{s_{i}^{\prime \prime}}\right) / /\left(\sqrt{t_{i}^{\prime}}, \sqrt{t_{i}^{\prime \prime}}\right) .
$$

Let $\left(\sqrt{s_{i}^{\prime}}, \sqrt{s_{i}^{\prime \prime}}\right)=\lambda\left(\sqrt{t_{i}^{\prime}}, \sqrt{t_{i}^{\prime \prime}}\right)$ for $\lambda>0$. Then we have

$$
\frac{u_{k}^{\prime \prime}}{u_{k}^{\prime}}=\frac{\left|u_{k}^{\prime \prime}\right|}{\left|u_{k}^{\prime}\right|}=\frac{\sqrt{s_{i}^{\prime \prime} t_{i}^{\prime \prime}}}{\sqrt{s_{i}^{\prime} t_{i}^{\prime}}}=\frac{\lambda t_{i}^{\prime \prime}}{\lambda t_{i}^{\prime}}=\frac{t_{i}^{\prime \prime}}{t_{i}^{\prime}}=\frac{s_{i}^{\prime \prime} / \lambda^{2}}{s_{i}^{\prime} / \lambda^{2}}=\frac{s_{i}^{\prime \prime}}{s_{i}^{\prime}} .
$$

Theorem 3.8. We have

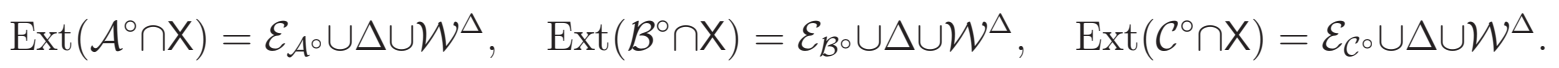

Proof. It suffices to show that every ray in $\mathcal{E}_{\mathcal{A}^{\circ}} \cup \Delta \cup \mathcal{W}^{\Delta}$ is extreme by Proposition 2.1. It is easy to see that diagonal states in $\Delta$ generate extreme rays of the convex cone $\mathcal{A}^{\circ} \cap \mathbf{X}$ by the conditions $W_{1}[1,4]$ and $W_{1}[2,3]$. For the remaining cases for $\mathcal{E}_{\mathcal{A}^{\circ}} \cup \mathcal{W}^{\Delta}$, we take $i=1,4$ and may assume that $W=\mathrm{X}(s, t, u)$ satisfies

$$
\sqrt{s_{1} t_{1}}=\left|u_{i}\right|>0 \quad \text { and } \quad s_{k}=t_{k}=u_{\ell}=0 \text { for } k \neq 1 \text { and } \ell \neq i .
$$

Suppose that $W=\mathrm{X}\left(s^{\prime}, t^{\prime}, u^{\prime}\right)+\mathrm{X}\left(s^{\prime \prime}, t^{\prime \prime}, u^{\prime \prime}\right)$ in $\mathcal{A}^{\circ} \cap \mathrm{X}$. For $k=2,3,4$, the condition $s_{k}=t_{k}=0$ implies $s_{k}^{\prime}=t_{k}^{\prime}=0=s_{k}^{\prime \prime}=t_{k}^{\prime \prime}$. Combining this with $W_{1}[2,3]$, we also have $u_{2}^{\prime}=u_{3}^{\prime}=0=u_{2}^{\prime \prime}=u_{3}^{\prime \prime}$. Applying Lemma 3.7 with $(i, j, k, \ell)=(1,4,1,4),(1,4,4,1)$, we get $\left(s^{\prime}, t^{\prime}, u^{\prime}\right) / /\left(s^{\prime \prime}, t^{\prime \prime}, u^{\prime \prime}\right)$. 


\section{Full Bi-SEPARABILITY AND BI-SEPARABILITY}

In this section, we consider convex cones $\mathcal{A} \cap \mathcal{B} \cap \mathcal{C}$ for full bi-separable states and $\mathcal{A}+\mathcal{B}+\mathcal{C}$ for bi-separable states, together with their dual cones $\mathcal{A}^{\circ}+\mathcal{B}^{\circ}+\mathcal{C}^{\circ}$ and $\mathcal{A}^{\circ} \cap \mathcal{B}^{\circ} \cap \mathcal{C}^{\circ}$, respectively. We first note that $\varrho=\mathrm{X}(a, b, z) \in \mathcal{A} \cap \mathcal{B} \cap \mathcal{C}$ if and only if $S_{1}[i, j]$ holds for every $i, j=1,2,3,4$, which is equivalent to the PPT condition of $\varrho$ [20, Theorem 5.3]. In order to find extreme rays of the cone $\mathcal{A} \cap \mathcal{B} \cap \mathcal{C} \cap \mathrm{X}$, we consider the condition

$$
S_{3}^{e}: \quad \sqrt{a_{i} b_{i}}=\left|z_{i}\right|=1, i=1,2,3,4,
$$

and define

$$
\mathcal{E}_{\mathcal{A} \cap \mathcal{B} \cap \mathcal{C}}=\left\{\varrho=\mathrm{X}(a, b, z): S_{3}^{e} \text { holds }\right\} .
$$

We also recall the inequality

$$
W_{3}: \quad \sum_{i=1}^{4} \sqrt{s_{i} t_{i}} \geq \sum_{i=1}^{4}\left|u_{i}\right|
$$

which appears in the characterization of decomposability of X-shaped entanglement witnesses in [20, Theorem 5.5].

Lemma 4.1. For a given self-adjoint $\mathrm{X}$-shaped matrix $W=\mathrm{X}(s, t, u)$, the following are equivalent.

(i) $\langle W, \varrho\rangle \geq 0$ for each $\varrho \in \mathcal{E}_{\mathcal{A} \cap \mathcal{B} \cap \mathcal{C}} \cup \Delta$;

(ii) $s_{i}, t_{i} \geq 0$ for $i=1,2,3,4$, and the inequality $W_{3}$ holds;

(iii) $\langle W, \varrho\rangle \geq 0$ for each $\varrho \in \mathcal{A} \cap \mathcal{B} \cap \mathcal{C}$.

Proof. For the direction (i) $\Longrightarrow$ (ii), we first obtain $s_{i}, t_{i} \geq 0$ from $\langle W, \varrho\rangle \geq 0$ for $\varrho \in \Delta$. In order to prove the inequality $W_{3}$, we may assume that $s_{i}, t_{i}>0$ as in the proof of Lemma 3.2. We can consider the state $\varrho$ defined by

$$
\varrho:=\mathrm{X}\left(\left(r_{1}, r_{2}, r_{3}, r_{4}\right),\left(r_{1}^{-1}, r_{2}^{-1}, r_{3}^{-1}, r_{4}^{-1}\right),\left(-e^{-\mathrm{i} \theta_{1}},-e^{-\mathrm{i} \theta_{2}},-e^{-\mathrm{i} \theta_{3}},-e^{-\mathrm{i} \theta_{4}}\right)\right)
$$

with $r_{i}=\sqrt{\frac{t_{i}}{s_{i}}}$ and $\theta_{k}=\arg u_{k}$. This state $\varrho$ belongs to $\mathcal{E}_{\mathcal{A} \cap \mathcal{B} \cap \mathcal{C}}$, and so $\langle W, \varrho\rangle \geq 0$ gives rise to the inequality $W_{3}$. For (ii) $\Longrightarrow$ (iii), it suffices to show that $\langle W, \varrho\rangle \geq 0$ for $\varrho=\mathrm{X}(a, b, z)$ satisfying $S_{1}[i, j]$ for all $i, j$ and $W=\mathrm{X}(s, t, u)$ satisfying $W_{3}$ by Corollary 2.3. Indeed, taking $M$ satisfying $\sqrt{a_{i} b_{i}} \geq M \geq\left|z_{j}\right|$ for each $i, j=1,2,3,4$, we have

$$
\sum_{i=1}^{4} \sqrt{s_{i} t_{i}} \sqrt{a_{i} b_{i}} \geq M \sum_{i=1}^{4} \sqrt{s_{i} t_{i}} \geq M \sum_{i=1}^{4}\left|u_{i}\right| \geq \sum_{i=1}^{4}\left|z_{i}\right|\left|u_{i}\right|,
$$

which implies $\langle W, \varrho\rangle \geq 0$, as in (하).

Since $(\mathcal{A} \cap \mathcal{B} \cap \mathcal{C})^{\circ}=\mathcal{A}^{\circ}+\mathcal{B}^{\circ}+\mathcal{C}^{\circ}$, the equivalence (ii) $\Longleftrightarrow$ (iii) in Lemma 4.1 gives another proof for [20, Theorem 5.5] which uses the duality principle. 
Proposition 4.2. [20, Theorem 5.5] An X-shaped self-adjoint matrix $W=\mathrm{X}(s, t, u)$ with nonnegative diagonals belongs to $\mathcal{A}^{\circ}+\mathcal{B}^{\circ}+\mathcal{C}^{\circ}$ if and only if the inequality $W_{3}$ holds.

For convex cones $C_{1}$ and $C_{2}$, it is clear that $\operatorname{Ext}\left(C_{1}+C_{2}\right) \subset \operatorname{Ext}\left(C_{1}\right) \cup \operatorname{Ext}\left(C_{2}\right)$ in general. Therefore, we see that $\operatorname{Ext}\left(\left(\mathcal{A}^{\circ}+\mathcal{B}^{\circ}+\mathcal{C}^{\circ}\right) \cap \mathrm{X}\right)$ is contained in the union of $\operatorname{Ext}\left(\mathcal{A}^{\circ} \cap \mathrm{X}\right)$, Ext $\left(\mathcal{B}^{\circ} \cap \mathrm{X}\right)$ and $\operatorname{Ext}\left(\mathcal{C}^{\circ} \cap \mathrm{X}\right)$ by Corollary 2.4. We show that they actually coincide.

Theorem 4.3. We have the following:

(i) $\operatorname{Ext}(\mathcal{A} \cap \mathcal{B} \cap \mathcal{C} \cap \mathrm{X})=\mathcal{E}_{\mathcal{A} \cap \mathcal{B} \cap \mathcal{C}} \cup \Delta$;

(ii) $\operatorname{Ext}\left(\left(\mathcal{A}^{\circ}+\mathcal{B}^{\circ}+\mathcal{C}^{\circ}\right) \cap \mathrm{X}\right)=\operatorname{Ext}\left(\mathcal{A}^{\circ} \cap \mathrm{X}\right) \cup \operatorname{Ext}\left(\mathcal{B}^{\circ} \cap \mathrm{X}\right) \cup \operatorname{Ext}\left(\mathcal{C}^{\circ} \cap \mathrm{X}\right)$.

Proof. For (i), it remains to show that every PPT state in $\mathcal{E}_{\mathcal{A} \cap \mathcal{B} \cap \mathcal{C}}$ generates an extreme ray of the cone $\mathcal{A} \cap \mathcal{B} \cap \mathcal{C}$. Suppose that $\varrho=\mathrm{X}(a, b, z)$ satisfies the condition $S_{3}^{e}$ and

$$
\varrho=\mathrm{X}\left(a^{\prime}, b^{\prime}, z^{\prime}\right)+\mathrm{X}\left(a^{\prime \prime}, b^{\prime \prime}, z^{\prime \prime}\right) \quad \text { in } \mathcal{A} \cap \mathcal{B} \cap \mathcal{C} \cap \mathrm{X} .
$$

Applying Lemma 3.4 with all the pairs $(i, j)$, we conclude $\left(a^{\prime}, b^{\prime}, z^{\prime}\right) / /\left(a^{\prime \prime}, b^{\prime \prime}, z^{\prime \prime}\right)$, and this completes the proof of (i).

In order to prove (ii), it suffices to show $\operatorname{Ext}\left(\mathcal{A}^{\circ} \cap \mathrm{X}\right) \subset \operatorname{Ext}\left(\left(\mathcal{A}^{\circ}+\mathcal{B}^{\circ}+\mathcal{C}^{\circ}\right) \cap \mathrm{X}\right)$. It is easy to see that diagonal states in $\Delta$ generate extreme rays in the convex cone $\left(\mathcal{A}^{\circ}+\mathcal{B}^{\circ}+\mathcal{C}^{\circ}\right) \cap \mathbf{X}$ by the condition $W_{3}$. We will show that $W=\mathrm{X}\left(r E_{1}, r^{-1} E_{1}, e^{\mathrm{i} \theta} E_{j}\right)$ generates an extreme ray of the cone $\left(\mathcal{A}^{\circ}+\mathcal{B}^{\circ}+\mathcal{C}^{\circ}\right) \cap \mathrm{X}$ for $j=1$, 4 . Suppose that

$$
W=\mathrm{X}\left(s^{\prime}, t^{\prime}, u^{\prime}\right)+\mathrm{X}\left(s^{\prime \prime}, t^{\prime \prime}, u^{\prime \prime}\right) \quad \text { in }\left(\mathcal{A}^{\circ}+\mathcal{B}^{\circ}+\mathcal{C}^{\circ}\right) \cap \mathrm{X} .
$$

For $i=2,3,4, s_{i}=0=t_{i}$ implies that $s_{i}^{\prime}=t_{i}^{\prime}=0=s_{i}^{\prime \prime}=t_{i}^{\prime \prime}$. By $W_{3}$, we have

$$
\begin{aligned}
1=\left|e^{\mathrm{i} \theta}\right|=\left|u_{j}^{\prime}+u_{j}^{\prime \prime}\right| \leq\left|u_{j}^{\prime}\right|+\left|u_{j}^{\prime \prime}\right| & \leq \sum_{k=1}^{4}\left(\left|u_{k}^{\prime}\right|+\left|u_{k}^{\prime \prime}\right|\right) \\
& \leq \sqrt{s_{1}^{\prime} t_{1}^{\prime}}+\sqrt{s_{1}^{\prime \prime} t_{1}^{\prime \prime}} \leq \sqrt{s_{1}^{\prime}+s_{1}^{\prime \prime}} \sqrt{t_{1}^{\prime}+t_{1}^{\prime \prime}}=1,
\end{aligned}
$$

and so it follows that $u_{k}^{\prime}=u_{k}^{\prime \prime}=0$ for $k \neq j$. Therefore, the summands in (7) belong to the cone $\mathcal{A}^{\circ} \cap \mathrm{X}$ by $W_{3}$ again, and we may apply Theorem 3.8.

Now, we turn our attention to the cone $\mathcal{A}^{\circ} \cap \mathcal{B}^{\circ} \cap \mathcal{C}^{\circ}$ and its dual cone. For each $i=1,2,3,4$, we consider the condition

$$
W_{3}^{e}[i]: \quad\left|u_{i}\right|=\sqrt{s_{j} t_{j}}=\sqrt{s_{k} t_{k}}=\sqrt{s_{\ell} t_{\ell}}=1, \quad \text { the others are zero, }
$$

where $j, k, \ell$ are chosen so that $i, j, k, \ell$ are mutually distinct, and define

$$
\mathcal{E}_{\mathcal{A}^{\circ} \cap \mathcal{B}^{\circ} \cap \mathcal{C}^{\circ}}=\left\{W=\mathrm{X}(s, t, u): W_{3}^{e}[i] \text { holds for some } i=1,2,3,4\right\} .
$$

We also consider the following inequality 


$$
S_{3}: \quad \sum_{j \neq i} \sqrt{a_{j} b_{j}} \geq\left|z_{i}\right|, \quad i=1,2,3,4
$$

These are exactly the inequalities which appear in the necessary criteria [16] for biseparability. We also refer to [17] for necessary criteria of multi-qubit bi-separable states. If $\varrho$ itself is X-shaped, then the converse is also true [18]. The authors have shown in [20, Corollary 3.4] that even a PPT mixture satisfies the multi-qubit analogue of $S_{3}$, to recover the above characterization of bi-separability of multi-qubit $\mathrm{X}$-states. We give here another alternative proof using the duality.

Lemma 4.4. For a given self-adjoint $\mathrm{X}$-shaped matrix $\varrho=\mathrm{X}(a, b, z)$, the following are equivalent.

(i) $\langle W, \varrho\rangle \geq 0$ for each $W \in \mathcal{E}_{\mathcal{A}^{\circ} \cap \mathcal{B}^{\circ} \cap \mathcal{C}^{\circ}} \cup \mathcal{W}^{\Delta} \cup \Delta$;

(ii) $\varrho$ is a state satisfying the inequality $S_{3}$;

(iii) $\langle W, \varrho\rangle \geq 0$ for each $W \in \mathcal{A}^{\circ} \cap \mathcal{B}^{\circ} \cap \mathcal{C}^{\circ}$.

Proof. For the direction (i) $\Longrightarrow$ (ii), we first note that $\varrho$ is a state as in the proof of Lemma 3.6. Now, we consider

$$
W:=X\left(\left(0, \sqrt{\frac{a_{2}}{b_{2}}}, \sqrt{\frac{a_{3}}{b_{3}}}, \sqrt{\frac{a_{4}}{b_{4}}},\right),\left(0, \sqrt{\frac{b_{2}}{a_{2}}}, \sqrt{\frac{b_{3}}{a_{3}}}, \sqrt{\frac{b_{4}}{a_{4}}},\right),\left(-e^{-\mathrm{i} \theta_{1}}, 0,0,0\right)\right),
$$

which belongs to $\mathcal{E}_{\mathcal{A}^{\circ} \cap \mathcal{B}^{\circ} \cap \mathcal{C}^{\circ}}$, where $\theta_{1}=\arg z_{1}$. Then, we have

$$
0 \leq \frac{1}{2}\langle\varrho, W\rangle=\sum_{j \neq 1} \sqrt{a_{j} b_{j}}-\left|z_{1}\right| \text {. }
$$

The other inequalities come out by the same way.

For the direction (ii) $\Longrightarrow$ (iii), it suffices to show the following:

$$
S_{3}, W_{1}[i, j] \text { for } i, j=1,2,3,4 \text { with } i \neq j \Longrightarrow\langle\mathbf{X}(s, t, u), \mathbf{X}(a, b, z)\rangle \geq 0
$$

by Corollary 2.3 and Proposition 3.3 . The inequality $\langle W, \varrho\rangle \geq 0$ is trivial when $W$ is positive, that is, $\sqrt{s_{i} t_{i}} \geq\left|u_{i}\right|$ for all $i=1,2,3,4$. Suppose that $W$ is not positive, and so there exists $i_{0} \in\{1,2,3,4\}$ such that $\sqrt{s_{i_{0}} t_{i_{0}}}<\left|u_{i_{0}}\right|$, say $\sqrt{s_{1} t_{1}}<\left|u_{1}\right|$ without loss of generality. We have

$$
\left(\sqrt{s_{1} t_{1}}+\sqrt{s_{i} t_{i}}\right) \sqrt{a_{i} b_{i}} \geq\left(\left|u_{1}\right|+\left|u_{i}\right|\right) \sqrt{a_{i} b_{i}}, \quad i=2,3,4,
$$

by $W_{1}[1, i]$. Summing up, we also have

$$
\begin{aligned}
\sqrt{s_{1} t_{1}} \sum_{i=2}^{4} \sqrt{a_{i} b_{i}}+\sum_{i=2}^{4} \sqrt{s_{i} t_{i}} \sqrt{a_{i} b_{i}} & \geq\left|u_{1}\right| \sum_{i=2}^{4} \sqrt{a_{i} b_{i}}+\sum_{i=2}^{4}\left|u_{i}\right| \sqrt{a_{i} b_{i}} \\
& \geq\left|u_{1}\right| \sum_{i=2}^{4} \sqrt{a_{i} b_{i}}+\sum_{i=2}^{4}\left|u_{i}\right|\left|z_{i}\right|
\end{aligned}
$$


which implies

$$
\begin{aligned}
\sum_{i=2}^{4}\left(\sqrt{s_{i} t_{i}} \sqrt{a_{i} b_{i}}-\left|u_{i}\right|\left|z_{i}\right|\right) & \geq\left(\left|u_{1}\right|-\sqrt{s_{1} t_{1}}\right) \sum_{i=2}^{4} \sqrt{a_{i} b_{i}} \\
& \geq\left(\left|u_{1}\right|-\sqrt{s_{1} t_{1}}\right)\left|z_{1}\right| \geq\left|u_{1}\right|\left|z_{1}\right|-\sqrt{s_{1} t_{1}} \sqrt{a_{1} b_{1}},
\end{aligned}
$$

by $S_{3}$ and $\sqrt{s_{1} t_{1}}<\left|u_{1}\right|$. Therefore, we have $\sum_{i=1}^{4} \sqrt{s_{i} t_{i}} \sqrt{a_{i} b_{i}} \geq \sum_{i=1}^{4}\left|u_{i}\right|\left|z_{i}\right|$, which completes the proof by (자).

Since the dual cone of $\mathcal{A}^{\circ} \cap \mathcal{B}^{\circ} \cap \mathcal{C}^{\circ}$ is just $\mathcal{A}+\mathcal{B}+\mathcal{C}$, we recover the following characterization of biseparability of three qubit states. Especially, every three qubit biseparable state with the $\mathrm{X}$-part $\mathrm{X}(a, b, z)$ must satisfy the inequalities $S_{3}$, as it was observed in [16].

Proposition 4.5. [16, 20, 18] For a three qubit $\mathrm{X}$-state $\varrho=\mathrm{X}(a, b, z)$, the following are equivalent:

(i) $\varrho$ belongs to $\mathcal{A}+\mathcal{B}+\mathcal{C}$;

(ii) the inequality $S_{3}$ holds.

As for extreme rays, we also have the following:

Theorem 4.6. We have the following:

(i) $\operatorname{Ext}((\mathcal{A}+\mathcal{B}+\mathcal{C}) \cap \mathrm{X})=\operatorname{Ext}(\mathcal{A} \cap \mathrm{X}) \cup \operatorname{Ext}(\mathcal{B} \cap \mathrm{X}) \cup \operatorname{Ext}(\mathcal{C} \cap \mathrm{X})$;

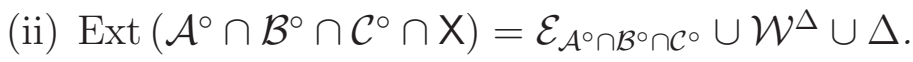

Proof. For (i), it suffices to show $\operatorname{Ext}(\mathcal{A} \cap \mathrm{X}) \subset \operatorname{Ext}((\mathcal{A}+\mathcal{B}+\mathcal{C}) \cap \mathrm{X})$. Suppose that $\varrho=\mathrm{X}(a, b, z)$ satisfies the condition $S_{1}^{e}[1,4]$, and

$$
\varrho=\mathrm{X}\left(a^{\prime}, b^{\prime}, z^{\prime}\right)+\mathrm{X}\left(a^{\prime \prime}, b^{\prime \prime}, z^{\prime \prime}\right) \quad \text { in }(\mathcal{A}+\mathcal{B}+\mathcal{C}) \cap \mathrm{X}
$$

Then we have $a_{i}^{\prime}=a_{i}^{\prime \prime}=b_{i}^{\prime}=b_{i}^{\prime \prime}=0$ for $i=2,3$, which also implies that $z_{i}^{\prime}=z_{i}^{\prime \prime}=0$ for $i=2,3$. By the inequality $S_{3}$, the summands in (8) must belong to the cone $\mathcal{A}$. Therefore, we can apply Theorem 3.5.

As for (ii), we note that matrices in $\Delta$ and $\mathcal{W}^{\Delta}$ generate extreme rays in $\mathcal{A}^{\circ} \cap \mathrm{X}$, and so they also generate extreme rays in the smaller cone $\mathcal{A}^{\circ} \cap \mathcal{B}^{\circ} \cap \mathcal{C}^{\circ} \cap \mathrm{X}$. Suppose that $W=\mathbf{X}(s, t, u)$ satisfies $W_{3}^{e}[1]$ and

$$
W=\mathrm{X}\left(s^{\prime}, t^{\prime}, u^{\prime}\right)+\mathrm{X}\left(s^{\prime \prime}, t^{\prime \prime}, u^{\prime \prime}\right) \quad \text { in } \mathcal{A}^{\circ} \cap \mathcal{B}^{\circ} \cap \mathcal{C}^{\circ} \cap \mathrm{X} .
$$

Applying Lemma 3.7 with $(i, j, k, \ell)=(2,1,1,2),(3,1,1,3),(4,1,1,4)$, we get $\left(s^{\prime}, t^{\prime}, u^{\prime}\right) / /$ $\left(s^{\prime \prime}, t^{\prime \prime}, u^{\prime \prime}\right)$.

It was shown in [20, Theorem 4.1] that $W \in \mathcal{E}_{\mathcal{A}^{\circ} \cap \mathcal{B}^{\circ} \cap \mathcal{C}^{\circ}}$ is an optimal genuine entanglement witness. This means that the set $\{\varrho \in \mathcal{P}:\langle W, \varrho\rangle<0\}$ of genuine entanglement detected by $W$ is maximal with respect to the inclusion. It is easy to see that extremeness implies optimality. We have shown in Theorem 4.6 that $W \in$ $\mathcal{E}_{\mathcal{A}^{\circ} \cap \mathcal{B}^{\circ} \cap \mathcal{C}^{\circ}}$ is extreme in the cone $\mathcal{A}^{\circ} \cap \mathcal{B}^{\circ} \cap \mathcal{C}^{\circ} \cap \mathrm{X}$. It would be interesting to ask if they are extreme in the much bigger convex cone $\mathcal{A}^{\circ} \cap \mathcal{B}^{\circ} \cap \mathcal{C}^{\circ}$. 


\section{INTERSECTIONS AND CONVEX HULLS OF TWO BASIC CONES}

In this section, we consider the following convex cones

$$
\mathcal{A} \cap \mathcal{B}, \quad \mathcal{B} \cap \mathcal{C}, \quad \mathcal{C} \cap \mathcal{A}, \quad \mathcal{A}+\mathcal{B}, \quad \mathcal{B}+\mathcal{C}, \quad \mathcal{C}+\mathcal{A},
$$

together with their dual cones:

$$
\mathcal{A}^{\circ}+\mathcal{B}^{\circ}, \quad \mathcal{B}^{\circ}+\mathcal{C}^{\circ}, \quad \mathcal{C}^{\circ}+\mathcal{A}^{\circ}, \quad \mathcal{A}^{\circ} \cap \mathcal{B}^{\circ}, \quad \mathcal{B}^{\circ} \cap \mathcal{C}^{\circ}, \quad \mathcal{C}^{\circ} \cap \mathcal{A}^{\circ}
$$

We look for inequalities characterizing the above convex cones, together with extreme rays of the cones. As for intersections of two cones, we just put together inequalities for both cones. For a three qubit $\mathrm{X}$-state $\varrho=\mathrm{X}(a, b, z)$, we have the following:

- $\varrho \in \mathcal{A} \cap \mathcal{B}$ if and only if $S_{1}[1,4], S_{1}[2,3], S_{1}[1,3], S_{1}[2,4]$ hold;

- $\varrho \in \mathcal{B} \cap \mathcal{C}$ if and only if $S_{1}[1,3], S_{1}[2,4], S_{1}[1,2], S_{1}[3,4]$ hold;

- $\varrho \in \mathcal{C} \cap \mathcal{A}$ if and only if $S_{1}[1,2], S_{1}[3,4], S_{1}[1,4], S_{1}[2,3]$ hold.

For an $\mathrm{X}$-shaped $W=\mathrm{X}(s, t, u)$, we also have

- $W \in \mathcal{A}^{\circ} \cap \mathcal{B}^{\circ}$ if and only if $W_{1}[1,4], W_{1}[2,3], W_{1}[1,3], W_{1}[2,4]$ hold;

- $W \in \mathcal{B}^{\circ} \cap \mathcal{C}^{\circ}$ if and only if $W_{1}[1,3], W_{1}[2,4], W_{1}[1,2], W_{1}[3,4]$ hold;

- $W \in \mathcal{C}^{\circ} \cap \mathcal{A}^{\circ}$ if and only if $W_{1}[1,2], W_{1}[3,4], W_{1}[1,4], W_{1}[2,3]$ hold.

In order to find extreme rays of the cones $\mathcal{B} \cap \mathcal{C} \cap \mathrm{X}, \mathcal{C} \cap \mathcal{A} \cap \mathrm{X}$ and $\mathcal{A} \cap \mathcal{B} \cap \mathrm{X}$, we consider the condition

$$
S_{2}^{e}[i, j]: \quad\left|z_{i}\right|=\sqrt{a_{i} b_{i}}=\sqrt{a_{k} b_{k}}=\sqrt{a_{\ell} b_{\ell}}=1, \quad \text { the others are zero, }
$$

for $i, j=1,2,3,4$ with $i \neq j$, where $k, \ell$ are chosen so that $i, j, k, \ell$ are mutually distinct. Here, we point out that $a_{j}=0=b_{j}$. We define

$$
\begin{aligned}
& \mathcal{E}_{\mathcal{B} \cap \mathcal{C}}=\left\{\varrho=\mathrm{X}(a, b, z): S_{2}^{e}[i, j] \text { holds for some }(i, j)=(1,4),(4,1),(2,3),(3,2)\right\}, \\
& \mathcal{E}_{\mathcal{C} \cap \mathcal{A}}=\left\{\varrho=\mathrm{X}(a, b, z): S_{2}^{e}[i, j] \text { holds for some }(i, j)=(1,3),(3,1),(2,4),(4,2)\right\}, \\
& \mathcal{E}_{\mathcal{A} \cap \mathcal{B}}=\left\{\varrho=\mathrm{X}(a, b, z): S_{2}^{e}[i, j] \text { holds for some }(i, j)=(1,2),(2,1),(3,4),(4,3)\right\},
\end{aligned}
$$

and consider the following inequalities

$$
W_{2}[i, j]: \quad \sum_{k \neq j} \sqrt{s_{k} t_{k}} \geq\left|u_{i}\right|, \quad \sum_{k \neq i} \sqrt{s_{k} t_{k}} \geq\left|u_{j}\right|
$$

for $i, j=1,2,3,4$ with $i \neq j$.

Lemma 5.1. For a given self-adjoint $\mathrm{X}$-shaped matrix $W=\mathrm{X}(s, t, u)$, the following are equivalent.

(i) $\langle W, \varrho\rangle \geq 0$ for each $\varrho \in \mathcal{E}_{\mathcal{B} \cap \mathcal{C}} \cup \mathcal{E}_{\mathcal{A} \cap \mathcal{B} \cap \mathcal{C}} \cup \Delta$ (respectively, $\mathcal{E}_{\mathcal{C} \cap \mathcal{A}} \cup \mathcal{E}_{\mathcal{A} \cap \mathcal{B} \cap \mathcal{C}} \cup \Delta$ and $\left.\mathcal{E}_{\mathcal{A} \cap \mathcal{B}} \cup \mathcal{E}_{\mathcal{A} \cap \mathcal{B} \cap \mathcal{C}} \cup \Delta\right)$

(ii) $s_{i}, t_{i} \geq 0$ for $i=1,2,3,4$ and the inequalities $W_{3}$ and $W_{2}[1,4], W_{2}[2,3]$ (respectively, $W_{2}[1,3], W_{2}[2,4]$ and $\left.W_{2}[1,2], W_{2}[3,4]\right)$ hold;

(iii) $\langle W, \varrho\rangle \geq 0$ for each $\varrho \in \mathcal{B} \cap \mathcal{C}$ (respectively, $\mathcal{C} \cap \mathcal{A}$ and $\mathcal{A} \cap \mathcal{B})$. 
Proof. The inequalities $s_{i}, t_{i} \geq 0$ and $W_{3}$ follow from Lemma 4.1. We will prove for $\mathcal{B} \cap \mathcal{C}$. The others follow by applying the operator $x_{A} \otimes x_{B} \otimes x_{C} \mapsto x_{\sigma(A)} \otimes x_{\sigma(B)} \otimes x_{\sigma(C)}$ for permutations $\sigma$ on $\{A, B, C\}$. To prove (i) $\Longrightarrow$ (ii), we may assume that all the diagonal elements $s_{i}$ and $t_{i}$ are nonzero, and consider four $\mathrm{X}$-states

$$
\begin{aligned}
& \varrho_{1,4}:=X\left(\left(\sqrt{\frac{t_{1}}{s_{1}}}, \sqrt{\frac{t_{2}}{s_{2}}}, \sqrt{\frac{t_{3}}{s_{3}}}, 0\right),\left(\sqrt{\frac{s_{1}}{t_{1}}}, \sqrt{\frac{s_{2}}{t_{2}}}, \sqrt{\frac{s_{3}}{t_{3}}}, 0\right),\left(-e^{-\mathrm{i} \theta_{1}}, 0,0,0\right)\right), \\
& \varrho_{2,3}:=X\left(\left(\sqrt{\frac{t_{1}}{s_{1}}}, \sqrt{\frac{t_{2}}{s_{2}}}, 0, \sqrt{\frac{t_{4}}{s_{4}}}\right),\left(\sqrt{\frac{s_{1}}{t_{1}}}, \sqrt{\frac{s_{2}}{t_{2}}}, 0, \sqrt{\frac{s_{4}}{t_{4}}}\right),\left(0,-e^{-\mathrm{i} \theta_{2}}, 0,0\right)\right), \\
& \varrho_{3,2}:=X\left(\left(\sqrt{\frac{t_{1}}{s_{1}}}, 0, \sqrt{\frac{t_{3}}{s_{3}}}, \sqrt{\frac{t_{4}}{s_{4}}}\right),\left(\sqrt{\frac{s_{1}}{t_{1}}}, 0, \sqrt{\frac{s_{3}}{t_{3}}}, \sqrt{\frac{s_{4}}{t_{4}}}\right),\left(0,0,-e^{-\mathrm{i} \theta_{3}}, 0\right)\right), \\
& \varrho_{4,1}:=X\left(\left(0, \sqrt{\frac{t_{2}}{s_{2}}}, \sqrt{\frac{t_{3}}{s_{3}}}, \sqrt{\frac{t_{4}}{s_{4}}}\right),\left(0, \sqrt{\frac{s_{2}}{t_{2}}}, \sqrt{\frac{s_{3}}{t_{3}}}, \sqrt{\frac{s_{4}}{t_{4}}}\right),\left(0,0,0,-e^{-\mathrm{i} \theta_{4}}\right)\right),
\end{aligned}
$$

with $\theta_{k}=\arg u_{k}$. These states belong to $\mathcal{E}_{\mathcal{B} \cap \mathcal{C}}$. We expand $\left\langle W, \varrho_{i, j}\right\rangle \geq 0$ to obtain $W_{2}[1,4]$ and $W_{2}[2,3]$.

For (ii) $\Longrightarrow$ (iii), it suffices to show $\langle W, \varrho\rangle \geq 0$ when $W=\mathbf{X}(s, t, u)$ satisfies $W_{2}[1,4]$, $W_{2}[2,3], W_{3}$, and $\varrho=\mathrm{X}(a, b, z)$ satisfies $S_{1}[1,3], S_{1}[2,4], S_{1}[1,2], S_{1}[3,4]$ by Corollary 2.3 and Proposition 3.1. If $\varrho \in \mathcal{A} \cap \mathcal{B} \cap \mathcal{C}$, then this is trivial by $W_{3}$ and Proposition 4.2. So, we may assume that $\varrho \notin \mathcal{A}$, especially $\left|z_{4}\right|>\sqrt{a_{1} b_{1}}$, without loss of generality. We begin with

$$
\begin{aligned}
\frac{1}{2}\langle W, \varrho\rangle \geq & \sum_{i=1}^{4}\left(\sqrt{s_{i} t_{i}} \sqrt{a_{i} b_{i}}-\left|u_{i}\right|\left|z_{i}\right|\right) \\
= & \left(\sqrt{s_{2} t_{2}} \sqrt{a_{2} b_{2}}+\sqrt{s_{3} t_{3}} \sqrt{a_{3} b_{3}}+\sqrt{s_{4} t_{4}} \sqrt{a_{4} b_{4}}-\left|u_{4}\right|\left|z_{4}\right|\right) \\
& +\left(\sqrt{s_{1} t_{1}} \sqrt{a_{1} b_{1}}-\left|u_{1}\right|\left|z_{1}\right|-\left|u_{2}\right|\left|z_{2}\right|-\left|u_{3}\right|\left|z_{3}\right|\right),
\end{aligned}
$$

as in (66). We have $\sqrt{a_{i} b_{i}} \geq\left|z_{4}\right|$ for $i=2,3,4$ by $S_{1}[2,4], S_{1}[3,4]$, and $\sqrt{a_{1} b_{1}} \geq\left|z_{i}\right|$ for $i=1,2,3$ by $S_{1}[1,2], S_{1}[1,3]$. By the inequality $W_{2}[1,4]$ and the assumption $\left|z_{4}\right|>\sqrt{a_{1} b_{1}}$, we have

$$
\begin{aligned}
\frac{1}{2}\langle W, \varrho\rangle & \geq\left(\sqrt{s_{2} t_{2}}+\sqrt{s_{3} t_{3}}+\sqrt{s_{4} t_{4}}-\left|u_{4}\right|\right)\left|z_{4}\right|+\left(\sqrt{s_{1} t_{1}}-\left|u_{1}\right|-\left|u_{2}\right|-\left|u_{3}\right|\right) \sqrt{a_{1} b_{1}} \\
& \geq\left(\sqrt{s_{2} t_{2}}+\sqrt{s_{3} t_{3}}+\sqrt{s_{4} t_{4}}-\left|u_{4}\right|\right) \sqrt{a_{1} b_{1}}+\left(\sqrt{s_{1} t_{1}}-\left|u_{1}\right|-\left|u_{2}\right|-\left|u_{3}\right|\right) \sqrt{a_{1} b_{1}} \\
& =\sqrt{a_{1} b_{1}}\left(\sum_{i=1}^{4} \sqrt{s_{i} t_{i}}-\sum_{i=1}^{4}\left|u_{i}\right|\right) .
\end{aligned}
$$

This is nonnegative by the inequality $W_{3}$, as it was desired.

By the equivalence (ii) $\Longleftrightarrow$ (iii), we have the following criteria for the convex hull of two basic dual cones:

Theorem 5.2. For a self-adjoint $W=\mathrm{X}(s, t, u)$ with nonnegative diagonals, we have the following:

(i) $W \in \mathcal{B}^{\circ}+\mathcal{C}^{\circ}$ if and only if $W_{2}[1,4], W_{2}[2,3]$ and $W_{3}$ hold; 
(ii) $W \in \mathcal{C}^{\circ}+\mathcal{A}^{\circ}$ if and only if $W_{2}[1,3], W_{2}[2,4]$ and $W_{3}$ hold;

(iii) $W \in \mathcal{A}^{\circ}+\mathcal{B}^{\circ}$ if and only if $W_{2}[1,2], W_{2}[3,4]$ and $W_{3}$ hold.

If $W$ is a self-adjoint three qubit matrix with the $\mathrm{X}$-part $\mathrm{X}(s, t, u)$, then the 'only if' parts hold.

Theorem 5.3. We have the following:

(i) $\operatorname{Ext}(\mathcal{B} \cap \mathcal{C} \cap \mathrm{X})=\mathcal{E}_{\mathcal{B} \cap \mathcal{C}} \cup \mathcal{E}_{\mathcal{A} \cap \mathcal{B} \cap \mathcal{C}} \cup \Delta$,

$\operatorname{Ext}(\mathcal{C} \cap \mathcal{A} \cap \mathrm{X})=\mathcal{E}_{\mathcal{C} \cap \mathcal{A}} \cup \mathcal{E}_{\mathcal{A} \cap \mathcal{B} \cap \mathcal{C}} \cup \Delta$,

$\operatorname{Ext}(\mathcal{A} \cap \mathcal{B} \cap \mathrm{X})=\mathcal{E}_{\mathcal{A} \cap \mathcal{B}} \cup \mathcal{E}_{\mathcal{A} \cap \mathcal{B} \cap \mathcal{C}} \cup \Delta$

(ii) $\operatorname{Ext}\left(\left(\mathcal{B}^{\circ}+\mathcal{C}^{\circ}\right) \cap \mathrm{X}\right)=\operatorname{Ext}\left(\mathcal{B}^{\circ} \cap \mathrm{X}\right) \cup \operatorname{Ext}\left(\mathcal{C}^{\circ} \cap \mathrm{X}\right)$,

$\operatorname{Ext}\left(\left(\mathcal{C}^{\circ}+\mathcal{A}^{\circ}\right) \cap \mathrm{X}\right)=\operatorname{Ext}\left(\mathcal{C}^{\circ} \cap \mathrm{X}\right) \cup \operatorname{Ext}\left(\mathcal{A}^{\circ} \cap \mathrm{X}\right)$,

$\operatorname{Ext}\left(\left(\mathcal{A}^{\circ}+\mathcal{B}^{\circ}\right) \cap \mathrm{X}\right)=\operatorname{Ext}\left(\mathcal{A}^{\circ} \cap \mathrm{X}\right) \cup \operatorname{Ext}\left(\mathcal{B}^{\circ} \cap \mathrm{X}\right)$.

Proof. (i). We will prove the first identity. Suppose that $\varrho=\mathrm{X}(a, b, z)$ satisfies $S_{2}^{e}[1,4]$ and

$$
\varrho=\mathrm{X}\left(a^{\prime}, b^{\prime}, z^{\prime}\right)+\mathrm{X}\left(a^{\prime \prime}, b^{\prime \prime}, z^{\prime \prime}\right) \quad \text { in } \mathcal{B} \cap \mathcal{C} \cap \mathrm{X} .
$$

The condition $a_{4}=b_{4}=0$ implies $a_{4}^{\prime}=b_{4}^{\prime}=z_{j}^{\prime}=0=a_{4}^{\prime \prime}=b_{4}^{\prime \prime}=z_{j}^{\prime \prime}$ for $j=$ $2,3,4$ by $S_{1}[2,4], S_{1}[3,4]$. Applying Lemma 3.4 with $(i, j)=(1,2)$ and $(1,3)$, we get $\left(a^{\prime}, b^{\prime}, z^{\prime}\right) / /\left(a^{\prime \prime}, b^{\prime \prime}, z^{\prime \prime}\right)$.

Next, suppose that $\varrho=\mathrm{X}(a, b, z)$ satisfies $S_{3}^{e}$ and

$$
\varrho=\mathrm{X}\left(a^{\prime}, b^{\prime}, z^{\prime}\right)+\mathrm{X}\left(a^{\prime \prime}, b^{\prime \prime}, z^{\prime \prime}\right) \quad \text { in } \mathcal{B} \cap \mathcal{C} \cap \mathrm{X} .
$$

Applying Lemma 3.4 with $(i, j)=(1,2),(2,1),(3,1),(4,2)$, we get $\left(a^{\prime}, b^{\prime}, z^{\prime}\right) / /\left(a^{\prime \prime}, b^{\prime \prime}, z^{\prime \prime}\right)$.

(ii). States in $\operatorname{Ext}\left(\mathcal{B}^{\circ} \cap \mathrm{X}\right) \cup \operatorname{Ext}\left(\mathcal{C}^{\circ} \cap \mathrm{X}\right)$ generate extreme rays of the convex cone $\left(\mathcal{A}^{\circ}+\mathcal{B}^{\circ}+\mathcal{C}^{\circ}\right) \cap \mathrm{X}$ by Theorem 4.3. Therefore, they also generate extreme rays in the smaller cone $\left(\mathcal{B}^{\circ}+\mathcal{C}^{\circ}\right) \cap \mathrm{X}$.

Now, we look for extreme rays of $\mathcal{B}^{\circ} \cap \mathcal{C}^{\circ} \cap \mathrm{X}$ (respectively, $\mathcal{C}^{\circ} \cap \mathcal{A}^{\circ} \cap \mathrm{X}$ and $\mathcal{A}^{\circ} \cap \mathcal{B}^{\circ} \cap \mathrm{X}$ ) to get conditions for the cone $\mathcal{B}+\mathcal{C}$ (respectively, $\mathcal{C}+\mathcal{A}$ and $\mathcal{A}+\mathcal{B}$ ). To do this, we consider the condition

$$
W_{2}^{e}[i, j]: \quad \sqrt{s_{i} t_{i}}=\sqrt{s_{j} t_{j}}=\left|u_{k}\right|=\left|u_{\ell}\right|=1, \quad \text { the others are zero, }
$$

for $i \neq j$, where $k$ and $\ell$ are chosen so that $i, j, k, \ell$ are mutually distinct, and define

$$
\begin{aligned}
& \mathcal{E}_{\mathcal{B}^{\circ} \cap \mathcal{C}^{\circ}}=\left\{W=\mathrm{X}(s, t, u): W_{2}^{e}[i, j] \text { holds for }(i, j)=(1,4) \text { or }(2,3)\right\}, \\
& \mathcal{E}_{\mathcal{C}^{\circ} \cap \mathcal{A}^{\circ}}=\left\{W=\mathrm{X}(s, t, u): W_{2}^{e}[i, j] \text { holds for }(i, j)=(1,3) \text { or }(2,4)\right\}, \\
& \mathcal{E}_{\mathcal{A}^{\circ} \cap \mathcal{B}^{\circ}}=\left\{W=\mathrm{X}(s, t, u): W_{2}^{e}[i, j] \text { holds for }(i, j)=(1,2) \text { or }(3,4)\right\} .
\end{aligned}
$$

We also consider the following inequalities

$$
S_{2}[i, j]: \quad \min \left\{\sqrt{a_{i} b_{i}}+\sqrt{a_{j} b_{j}}, \sqrt{a_{k} b_{k}}+\sqrt{a_{\ell} b_{\ell}}\right\} \geq \max \left\{\left|z_{i}\right|+\left|z_{j}\right|,\left|z_{k}\right|+\left|z_{\ell}\right|\right\},
$$


for $i \neq j$, where $k, \ell$ are chosen so that $i, j, k, \ell$ are mutually distinct.

These inequalities have been used in [11] to get necessary conditions for a three state $\varrho$ with the $\mathrm{X}$-part $\mathrm{X}(a, b, z)$ to belong to $\mathcal{B}+\mathcal{C}, \mathcal{C}+\mathcal{A}$ and $\mathcal{A}+\mathcal{B}$ respectively. We show in Theorem 5.5 that they provide actually sufficient conditions when $\varrho$ itself X-shaped. Note that

- $S_{2}[1,4]$ holds if and only if $S_{2}[2,3]$ holds;

- $S_{2}[1,3]$ holds if and only if $S_{2}[2,4]$ holds;

- $S_{2}[1,2]$ holds if and only if $S_{2}[3,4]$ holds.

Lemma 5.4. For a given self-adjoint $\mathrm{X}$-shaped matrix $\varrho=\mathrm{X}(a, b, z)$, the following are equivalent.

(i) $\langle W, \varrho\rangle \geq 0$ for each $W \in \mathcal{E}_{\mathcal{B}^{\circ} \cap \mathcal{C}^{\circ}} \cup \mathcal{W}^{\Delta} \cup \Delta$ (respectively, $\mathcal{E}_{\mathcal{C}^{\circ} \cap \mathcal{A}^{\circ}} \cup \mathcal{W}^{\Delta} \cup \Delta$ and $\left.\mathcal{E}_{\mathcal{A}^{\circ} \cap \mathcal{B}^{\circ}} \cup \mathcal{W}^{\Delta} \cup \Delta\right)$

(ii) @ is a state satisfying the inequalities $S_{2}[1,4]$ (respectively, $S_{2}[1,3]$ and $S_{2}[1,2]$ ) hold;

(iii) $\langle W, \varrho\rangle \geq 0$ for each $W \in \mathcal{B}^{\circ} \cap \mathcal{C}^{\circ}\left(\right.$ respectively, $\mathcal{C}^{\circ} \cap \mathcal{A}^{\circ}$ and $\left.\mathcal{A}^{\circ} \cap \mathcal{B}^{\circ}\right)$.

Proof. Although the proof of the direction (i) $\Longrightarrow$ (ii) already appears in [11, we include it here for the completeness. We consider $\mathbf{X}$-shaped three qubit self-adjoint matrices

$$
\begin{aligned}
W & :=\mathrm{X}\left(\left(\sqrt{\frac{b_{1}}{a_{1}}}, 0,0, \sqrt{\frac{b_{4}}{a_{4}}}\right),\left(\sqrt{\frac{a_{1}}{b_{1}}}, 0,0, \sqrt{\frac{a_{4}}{b_{4}}}\right),\left(0,-e^{-\mathrm{i} \theta_{2}},-e^{-\mathrm{i} \theta_{3}}, 0\right)\right) \\
W^{\prime} & :=\mathrm{X}\left(\left(0, \sqrt{\frac{b_{2}}{a_{2}}}, \sqrt{\frac{b_{3}}{a_{3}}}, 0\right),\left(0, \sqrt{\frac{a_{2}}{b_{2}}}, \sqrt{\frac{a_{3}}{b_{3}}}, 0\right),\left(-e^{-\mathrm{i} \theta_{1}}, 0,0,-e^{-\mathrm{i} \theta_{4}}\right)\right),
\end{aligned}
$$

for $\theta_{i}=\arg z_{i}$. Then, both $W$ and $W^{\prime}$ belong to $\mathcal{E}_{\mathcal{B}^{\circ} \cap \mathcal{C}^{\circ}}$. We have

$$
\begin{aligned}
& 0 \leq \frac{1}{2}\langle\varrho, W\rangle=\sqrt{a_{1} b_{1}}+\sqrt{a_{4} b_{4}}-\left|z_{2}\right|-\left|z_{3}\right|, \\
& 0 \leq \frac{1}{2}\left\langle\varrho, W^{\prime}\right\rangle=\sqrt{a_{2} b_{2}}+\sqrt{a_{3} b_{3}}-\left|z_{1}\right|-\left|z_{4}\right| .
\end{aligned}
$$

For the implication (ii) $\Longrightarrow$ (iii), suppose that $\varrho=\mathrm{X}(a, b, z)$ satisfies $S_{2}[1,4]$. By Corollary 2.3. it suffices to show $\langle W, \varrho\rangle \geq 0$ for every $W=\mathrm{X}(s, t, u) \in \mathcal{B}^{\circ} \cap \mathcal{C}^{\circ}$. This is trivial when $\mathbf{X}(s, t, u)$ is positive, that is, $\sqrt{s_{i} t_{i}} \geq\left|u_{i}\right|$ for all $i=1,2,3,4$. We may assume without loss of generality that

$$
0>\sqrt{s_{1} t_{1}}-\left|u_{1}\right|=\min \left\{\sqrt{s_{i} t_{i}}-\left|u_{i}\right|: i=1,2,3,4\right\} .
$$

Then we have

$$
\begin{aligned}
&\left(\sqrt{s_{1} t_{1}}+\sqrt{s_{2} t_{2}}\right) \sqrt{a_{2} b_{2}} \geq\left(\left|u_{1}\right|+\left|u_{2}\right|\right) \sqrt{a_{2} b_{2}}, \\
&\left(\sqrt{s_{1} t_{1}}+\sqrt{s_{3} t_{3}}\right) \sqrt{a_{3} b_{3}} \geq\left(\left|u_{1}\right|+\left|u_{3}\right|\right) \sqrt{a_{3} b_{3}}, \\
&\left(\left|u_{1}\right|+\sqrt{s_{4} t_{4}}\right)\left|z_{4}\right| \geq\left(\sqrt{s_{1} t_{1}}+\left|u_{4}\right|\right)\left|z_{4}\right|, \\
& 19
\end{aligned}
$$


where the first and second inequalities follow from $W_{1}[1,2]$ and $W_{1}[1,3]$, respectively, and the last one comes out from the equality in (9). Put $M=\sqrt{a_{2} b_{2}}+\sqrt{a_{3} b_{3}}-\left|z_{4}\right|$. Summing up the above three inequalities, we have

$$
\sqrt{s_{1} t_{1}} M+\sum_{i=2}^{3} \sqrt{s_{i} t_{i}} \sqrt{a_{i} b_{i}}+\sqrt{s_{4} t_{4}}\left|z_{4}\right| \geq\left|u_{1}\right| M+\sum_{i=2}^{3}\left|u_{i}\right| \sqrt{a_{i} b_{i}}+\left|u_{4}\right|\left|z_{4}\right|,
$$

which implies

$$
\sqrt{s_{1} t_{1}} M+\sum_{i=2}^{4} \sqrt{s_{i} t_{i}} \sqrt{a_{i} b_{i}} \geq\left|u_{1}\right| M+\sum_{i=2}^{4}\left|u_{i}\right|\left|z_{i}\right| .
$$

Because $M \geq\left|z_{1}\right|$ by $S_{2}[1,4]$ and $\left|u_{1}\right|-\sqrt{s_{1} t_{1}}>0$ by (9), we have

$$
\begin{aligned}
\sum_{i=2}^{4}\left(\sqrt{s_{i} t_{i}} \sqrt{a_{i} b_{i}}-\left|u_{i}\right|\left|z_{i}\right|\right) & \geq\left(\left|u_{1}\right|-\sqrt{s_{1} t_{1}}\right) M \\
& \geq\left(\left|u_{1}\right|-\sqrt{s_{1} t_{1}}\right)\left|z_{1}\right| \geq\left|u_{1}\right|\left|z_{1}\right|-\sqrt{s_{1} t_{1}} \sqrt{a_{1} b_{1}} .
\end{aligned}
$$

This gives $\sum_{i=1}^{4} \sqrt{s_{i} t_{i}} \sqrt{a_{i} b_{i}} \geq \sum_{i=1}^{4}\left|u_{i}\right|\left|z_{i}\right|$, and $\langle W, \varrho\rangle \geq 0$ by by ([6).

Because $\mathcal{B}+\mathcal{C}$ (respectively, $\mathcal{C}+\mathcal{A}$ and $\mathcal{A}+\mathcal{B}$ ) is the dual of $\mathcal{B}^{\circ} \cap \mathcal{C}^{\circ}$ (respectively, $\mathcal{C}^{\circ} \cap \mathcal{A}^{\circ}$ and $\mathcal{A}^{\circ} \cap \mathcal{B}^{\circ}$ ), the equivalence between (ii) and (iii) of Lemma 5.4 gives rise to the following characterization of the cone $\mathcal{B}+\mathcal{C}$ (respectively, $\mathcal{C}+\mathcal{A}$ and $\mathcal{A}+\mathcal{B}$ ) for X-states.

Theorem 5.5. For a three qubit $\mathrm{X}$-state $\varrho=\mathrm{X}(a, b, z)$, we have the following:

(i) $\varrho \in \mathcal{B}+\mathcal{C}$ if and only if $S_{2}[1,4]$ holds;

(ii) $\varrho \in \mathcal{C}+\mathcal{A}$ if and only if $S_{2}[1,3]$ holds;

(iii) $\varrho \in \mathcal{A}+\mathcal{B}$ if and only if $S_{2}[1,2]$ holds.

For a general three qubit state @ with the $\mathbf{X}$-part $\mathbf{X}(a, b, z)$, the 'only if' parts hold.

Theorem 5.6. We have the following:

(i) $\operatorname{Ext}\left(\mathcal{B}^{\circ} \cap \mathcal{C}^{\circ} \cap \mathrm{X}\right)=\mathcal{E}_{\mathcal{B}^{\circ} \cap \mathcal{C}^{\circ}} \cup \mathcal{W}^{\Delta} \cup \Delta$,

$\operatorname{Ext}\left(\mathcal{C}^{\circ} \cap \mathcal{A}^{\circ} \cap \mathrm{X}\right)=\mathcal{E}_{\mathcal{C}^{\circ} \cap \mathcal{A}^{\circ}} \cup \mathcal{W}^{\Delta} \cup \Delta$

$\operatorname{Ext}\left(\mathcal{A}^{\circ} \cap \mathcal{B}^{\circ} \cap \mathrm{X}\right)=\mathcal{E}_{\mathcal{A}^{\circ} \cap \mathcal{B}^{\circ}} \cup \mathcal{W}^{\Delta} \cup \Delta$

(ii) $\operatorname{Ext}((\mathcal{B}+\mathcal{C}) \cap \mathrm{X})=\operatorname{Ext}(\mathcal{B} \cap \mathrm{X}) \cup \operatorname{Ext}(\mathcal{C} \cap \mathrm{X})$,

$\operatorname{Ext}((\mathcal{C}+\mathcal{A}) \cap \mathrm{X})=\operatorname{Ext}(\mathcal{C} \cap \mathrm{X}) \cup \operatorname{Ext}(\mathcal{A} \cap \mathrm{X})$,

$\operatorname{Ext}((\mathcal{A}+\mathcal{B}) \cap \mathrm{X})=\operatorname{Ext}(\mathcal{A} \cap \mathrm{X}) \cup \operatorname{Ext}(\mathcal{B} \cap \mathrm{X})$.

Proof. (i). We will prove the first identity. Since elements in $\Delta$ and $\mathcal{W}^{\Delta}$ are extremal in $\mathcal{B}^{\circ} \cap \mathrm{X}$, they are also extremal in the smaller cone $\mathcal{B}^{\circ} \cap \mathcal{C}^{\circ} \cap \mathrm{X}$. Suppose that $W=\mathbf{X}(s, t, u)$ satisfies $W_{2}^{e}[1,4]$ and

$$
W=\mathrm{X}\left(s^{\prime}, t^{\prime}, u^{\prime}\right)+\mathrm{X}\left(s^{\prime \prime}, t^{\prime \prime}, u^{\prime \prime}\right) \quad \text { in } \mathcal{B}^{\circ} \cap \mathcal{C}^{\circ} \cap \mathrm{X} .
$$

Applying Lemma 3.7 with $(i, j, k, \ell)=(1,2,2,1),(4,2,2,4),(1,3,3,1),(4,3,3,4)$, we get $\left(s^{\prime}, t^{\prime}, u^{\prime}\right) / /\left(s^{\prime \prime}, t^{\prime \prime}, u^{\prime \prime}\right)$. 


\begin{tabular}{|c|c|c|c|c|c|}
\hline criteria & extreme & states & witnesses & extreme & criteria \\
\hline$\overline{S_{1}[1,4],}$ & $\overline{S_{1}^{e}[1,4]}$ & \multirow{4}{*}{$\mathcal{A} \cap \mathrm{X}$} & \multirow{4}{*}{$\mathcal{A}^{\circ} \cap \mathrm{X}$} & $\overline{W_{1}^{e}[1,4]}$ & $\overline{W_{1}[1,4]}$ \\
\hline & & & & $W_{1}^{e}[4,1]$ & \\
\hline$S_{1}[2,3]$ & $S_{1}^{c}[2,3]$ & & & $\begin{array}{l}W_{1}^{e}[2,3] \\
W_{1}^{e}[3,2]\end{array}$ & $W_{1}[2,3]$ \\
\hline Prop.3.1 & Th.3.5 & & & Th.3.8 & Prop.3.3 \\
\hline$S_{1}[i, j]$ & $S_{3}^{e}$ & \multirow[t]{2}{*}{$\mathcal{A} \cap \mathcal{B} \cap \mathcal{C} \cap \mathrm{X}$} & \multirow[t]{2}{*}{$\left(\mathcal{A}^{\circ}+\mathcal{B}^{\circ}+\mathcal{C}^{\circ}\right) \cap \mathrm{X}$} & $W_{1}^{e}[i, j]$ & $W_{3}$ \\
\hline Prop.3.1 & Th.4.3(i) & & & Th.4.3(ii) & Prop. 4.2 \\
\hline$S_{3}$ & $S_{1}^{e}[i, j]$ & \multirow[t]{2}{*}{$(\mathcal{A}+\mathcal{B}+\mathcal{C}) \cap \mathrm{X}$} & \multirow[t]{2}{*}{$\mathcal{A}^{\circ} \cap \mathcal{B}^{\circ} \cap \mathcal{C}^{\circ} \cap \mathrm{X}$} & $W_{3}^{e}[i]$ & $W_{1}[i, j]$ \\
\hline Prop.4.5 & Th.4.6(i) & & & Th.4.6(ii) & Prop. 3.3 \\
\hline$S_{1}[1,4]$ & $S_{3}^{e}$ & \multirow{8}{*}{$\mathcal{A} \cap \mathcal{B} \cap \mathrm{X}$} & \multirow{8}{*}{$\left(\mathcal{A}^{\circ}+\mathcal{B}^{\circ}\right) \cap \mathrm{X}$} & $W_{1}^{e}[1,4]$ & $W_{3}$ \\
\hline & & & & $W_{1}^{e}[4,1]$ & \\
\hline$S_{1}[2,3]$ & $S_{2}^{e}[1,2]$ & & & $W_{1}^{e}[2,3]$ & $W_{2}[1,2]$ \\
\hline \multirow{3}{*}{$S_{1}[1,3]$} & $S_{2}^{e}[2,1]$ & & & $W_{1}^{e}[3,2]$ & \multirow{5}{*}{$W_{2}[3,4]$} \\
\hline & & & & $W_{1}^{e}[1,3]$ & \\
\hline & $S_{2}^{e}[3,4]$ & & & $W_{1}^{e}[3,1]$ & \\
\hline$S_{1}[2,4]$ & $S_{2}^{e}[4,3]$ & & & $\begin{array}{l}W_{1}^{e}[2,4] \\
W^{e}[4, ?]\end{array}$ & \\
\hline Prop.3.1 & Th.5.3(i) & & & Th.5.3(ii) & \\
\hline \multirow{5}{*}{$S_{2}[1,2]$} & $S_{1}^{e}[1,4]$ & \multirow{5}{*}{$(\mathcal{A}+\mathcal{B}) \cap \mathrm{X}$} & \multirow{5}{*}{$\mathcal{A}^{\circ} \cap \mathcal{B}^{\circ} \cap \mathrm{X}$} & & $W_{1}[1,4]$ \\
\hline & $S_{1}^{e}[2,3]$ & & & $W_{2}^{e}[1,2]$ & $W_{1}[2,3]$ \\
\hline & $S_{1}^{e}[1,3]$ & & & $W_{2}^{e}[3,4]$ & $W_{1}[1,3]$ \\
\hline & $S_{1}^{e}[2,4]$ & & & & $W_{1}[2,4]$ \\
\hline & Th.5.6(ii) & & & Th.5.6(i) & Prop. 3.3 \\
\hline
\end{tabular}

TABLE 1. Criteria and extreme rays of convex cones: Conditions for 'criteria' and 'extreme' are connected by 'and' and 'or', respectively.

(ii). Since states in $\operatorname{Ext}(\mathcal{B} \cap \mathbf{X}) \cup \operatorname{Ext}(\mathcal{C} \cap \mathbf{X})$ are extremal in the convex cone $(\mathcal{A}+\mathcal{B}+\mathcal{C}) \cap \mathrm{X}$ by Theorem 4.6, they are also extremal in the smaller cone $(\mathcal{B}+\mathcal{C}) \cap \mathrm{X}$.

\section{Summary}

In this paper, we have considered the convex cones in the diagrams (3) and (44) arising from classification of partial separability/entanglement of three qubit states and their witnesses. For those convex cones, we got the following results:

- characterization for X-shaped matrices by algebraic inequalities, which give rise to necessary criteria for general three qubit states/witnesses in terms of diagonal and anti-diagonal entries;

- finding all the extreme rays of the cones consisting of $\mathrm{X}$-shaped matrices, with which we may exhibit all X-shaped matrices in the cones.

We summarize the results in Table 1 . We note our characterizion is one of very few cases when we may check separability by inequalities, without decomposing into the sum of pure product states. For example, we may check separability for $2 \otimes 2$ and $2 \otimes 3$ 
cases by the PPT condition. We may also check full separability of multi-qubit $\mathbf{X}$-states by inequalities [14, 15, 12, Checking separability with inequalities in this paper was possible through the duality and characterizing extreme rays of the dual cones.

This work has been partly motivated by the questions [10] on the existence of states in the seven classes arising in the classification of partial entanglement, including the following classes:

$$
\begin{aligned}
\mathcal{C}^{2,6,1} & :=\mathcal{A} \cap(\mathcal{B}+\mathcal{C}) \cap \mathcal{B}^{\mathrm{c}} \cap \mathcal{C}^{\mathrm{c}} \\
\mathcal{C}^{2,4} & :=(\mathcal{A}+\mathcal{B}) \cap(\mathcal{B}+\mathcal{C}) \cap(\mathcal{C}+\mathcal{A}) \cap \mathcal{A}^{\mathrm{c}} \cap \mathcal{B}^{\mathrm{c}} \cap \mathcal{C}^{\mathrm{c}}, \\
\mathcal{C}^{2,3,1} & :=(\mathcal{A}+\mathcal{B}) \cap(\mathcal{C}+\mathcal{A}) \cap \mathcal{A}^{\mathrm{c}} \cap(\mathcal{B}+\mathcal{C})^{\mathrm{c}}
\end{aligned}
$$

together with the convex cones obtained by permuting systems. Here, $\mathcal{C}^{2,6,1}, \mathcal{C}^{2,4}$ and $\mathcal{C}^{2,3,1}$ are notations in [10]. The authors [11] gave examples of $\mathbf{X}$-shaped states belonging to those classes. In this paper, we gave complete necessary and sufficient conditions for $\mathrm{X}$-states to be members of the classes. For example, an $\mathrm{X}$-state $\varrho=\mathrm{X}(a, b, z)$ belongs to the class $\mathcal{C}^{2,6,1}$ if and only if the following hold:

- $\varrho$ satisfies the inequalities $S_{1}[1,4], S_{1}[2,3]$ and $S_{2}[1,4]$;

- $\varrho$ violates $S_{1}[1,3]$ or $S_{1}[2,4]$;

- $\varrho$ violates $S_{1}[1,2]$ or $S_{1}[3,4]$.

The example $\varrho=\mathrm{X}((0,1,1,2),(0,1,1,2),(0,1,1,0))$ given in [1] satisfies $S_{1}[1,4]$, $S_{1}[2,3]$ and $S_{2}[1,4]$, but violates $S_{1}[1,3]$ and $S_{1}[1,2]$.

It is natural to ask what happens in the four qubit system, or arbitrary qubit systems. We began with the result [20] that an X-shaped multi-qubit state is separable with respect to a bi-partition of systems if and only if it is of positive partial transpose with respect to the same bi-partition. This was crucial to give characterizations in terms of diagonal entries and the modulus of anti-diagonal entries. But this is not the case for tri-partitions. In the three qubit system, considering tri-partition is amount to full separability. We need the phase parts, that is, the angular parts of anti-diagonal entries, as well as the modulus parts to characterize full separability of three qubit X-states. See [14, 15, 13]. We note that all kinds of partial separability come out from bi-partitions in the three qubit case. But, it is necessary to consider tri-partitions as well as bi-partitions in the four qubit case. See [9, 25]. Therefore, exploring partial separability/entanglement in general qubit system must be a very challenging project even for X-shaped states.

\section{REFERENCES}

[1] C. H. Bennett, D. P. DiVincenzo, T. Mor, P. W. Shor, J. A. Smolin and B. M. Terhal, Unextendible product bases and bound entanglement, Phys. Rev. Lett. 82 (1999), 5385-5388.

[2] A. Acin, D. Bruß, M. Lewenstein and A. Sanpera, Classification of mixed three-qubit states, Phys. Rev. Lett. 87 (2001), 040401.

[3] V. Coffman, J. Kundu and W. K. Wootters, Distributed entanglement, Phys. Rev. A 61 (2000), 052306 . 
[4] W. Dür and J. I. Cirac, Classification of multi-qubit mixed states: separability and distillability properties, Phys. Rev. A 61 (2000), 042314.

[5] W. Dür, J. I. Cirac and R. Tarrach, Separability and Distillability of Multiparticle Quantum Systems, Phys. Rev. Lett. 83 (1999), 3562-3565.

[6] W. Dür, G. Vidal and J. I. Cirac, Three qubits can be entangled in two inequivalent ways, Phys. Rev. A 62 (2000), 062314.

[7] M. Seevinck and J. Uffink, Partial separability and etanglement criteria for multiqubit quantum states, Phys. Rev. A 78 (2008), 032101.

[8] S. Szalay, Separability criteria for mixed three-qubit states, Phys. Rev. A 83 (2011), 062337.

[9] S. Szalay, Multipartite entanglement measures, Phys. Rev. A 92 (2015), 042329.

[10] S. Szalay and Z. Kökényesi, Partial separability revisited: Necessary and sufficient criteria, Phys. Rev. A 86 (2012), 032341.

[11] K. H. Han and S.-H. Kye, Construction of three-qubit biseparable states distinguishing kinds of entanglement in a partial separability classification, Phys. Rev. A, 99 (2019), 032304.

[12] K. H. Han and S.-H, Kye, Separability of three qubit Greenberger-Horne-Zeilinger diagonal states, J. Phys. A: Math. Theor. 50 (2017), 145303.

[13] K. H. Han and S.-H, Kye, The role of phases in detecting three qubit entanglement, J. Math. Phys. 58 (2017), 102201.

[14] L. Chen, K. H. Han and S.-H, Kye, Separability criterion for three-qubit states with a four dimensional norm, J. Phys. A: Math. Theor. 50 (2017) 345303.

[15] K.-C. Ha, K. H. Han and S.-H. Kye, Separability of multi-qubit states in terms of diagonal and anti-diagonal entries, Quantum Inf. Process. 18 (2019), 34.

[16] O. Gühne and M. Seevinck, Separability criteria for genuine multiparticle entanglement, New J. Phys. 12 (2010), 053002.

[17] T. Gao and Y. Hong, Separability criteria for several classes of n-partite quantum states, Eur. Phys. J. D 61 (2011), 765-771.

[18] S. M. H. Rafsanjani, M. Huber, C. J. Broadbent and J. H. Eberly, Genuinely multipartite concurrence of $N$-qubit X matrices, Phys. Rev. A 86 (2012), 062303.

[19] K. H. Han and S.-H, Kye, Various notions of positivity for bi-linear maps and applications to tri-partite entanglement, J. Math. Phys. 57 (2016), 015205.

[20] K. H. Han and S.-H, Kye, Construction of multi-qubit optimal genuine entanglement witnesses, J. Phys. A: Math. Theor. 49 (2016), 175303.

[21] B. M. Terhal, Bell Inequalities and the Separability Criterion, Phys. Lett. A 271 (2000), 319-326.

[22] M.-H. Eom and S.-H. Kye, Duality for positive linear maps in matrix algebras, Math. Scand. 86 (2000), 130-142.

[23] R. T. Rockafellar, Convex Analysis, Princeton Univ. Press, 1970.

[24] D. M. Greenberger, M. A. Horne and A. Zeilinger, Going beyond Bell's theorem, in Kafatos M. (eds) Bell's Theorem, Quantum Theory and Conceptions of the Universe. Fundamental Theories of Physics, Vol. 37, Springer, Dordrecht, 1989.

[25] S. Szalay, The classification of multipartite quantum correlation, J. Phys. A: Math. Theor. 51 (2018), 485302 .

Kyung Hoon Han, Department of Data Science, The University of Suwon, GyeonggiDO 445-743, KOREA

E-mail address: kyunghoon.han at gmail.com

Seung-Hyeok Kye, Department of Mathematics and Institute of Mathematics, Seoul

National University, Seoul 151-742, Korea

E-mail address: kye at snu.ac.kr 